\title{
Advanced RF/Baseband Interconnect Schemes for Inter- and Intra-ULSI Communications
}

\author{
Mau-Chung Frank Chang, Fellow, IEEE, Ingrid Verbauwhede, Senior Member, IEEE, Charles Chien, Member, IEEE, \\ Zhiwei Xu, Jongsun Kim, Student Member, IEEE, Jenwei Ko, Qun Gu, and Bo-Cheng Lai
}

Invited Paper

\begin{abstract}
Future inter- and intra-ULSI interconnect systems demand extremely high data rates (up to $100 \mathrm{Gbps} / \mathrm{pin}$ or 20-Tbps aggregate) as well as bidirectional multiI/O concurrent service, re-configurable computing/processing architecture, and total compatibility with mainstream silicon system-on-chip and system-in-package technologies. In this paper, we review recent advances in interconnect schemes that promise to meet all of the above system requirements. Unlike traditional wired interconnects based solely on time-division multiple access for data transmission, these new interconnect schemes facilitate the use of additional multiple access techniques including code-division multiple access and frequency-division multiple access to greatly increase bandwidth and channel concurrency as well as to reduce channel latency. The physical transmission line is no longer limited to a direct-coupled metal wire. Rather, it can be accomplished via either wired or wireless mediums through capacitor couplers that reduce the baseband noise and de power consumption while simplifying the fabrication process by eliminating vertical metal studs needed in three-dimensional ICs. These new advances in interconnect schemes would fundamentally alter the paradigm of ULSI data communications and enable the design of next-generation computing/processing systems.
\end{abstract}

Index Terms-3-D IC interconnect, CDMA DRAM, CDMA-interconnect (CDMA-I), FDMA-interconnect (FDMA-I), FDMA DRAM, inter/intra-ULSI communications, multilevel signal clock data recovery, multicarrier DCMA-Interconnect (MCCDMA-I), realtime re-configurable interconnects, RF-interconnect (RF-I), re-configurable interconnect for next-generation systems (RINGS), simultaneous and bidirectional multiI/O service.

\section{INTRODUCTION}

$\mathbf{T}$ HE SYSTEM performance of modern ULSI is being limited by its interconnect bandwidth in both on-chip and chip-to-chip communications [1]-[3]. The rapid evolution of ULSI has demanded that the interconnect system be fast and at the same time be flexible, reliable, and low cost. Ideally, future interconnect systems must encompass the following important features:

- Ultra-high data rates (e.g., $>100 \mathrm{Gbps} /$ pin or 20-Tbps aggregate [2], defined as the total sum of data rate for each pin on a chip or within a system of chips)

Manuscript received September 8, 2004. The review of this paper was arranged by Editor B. Zhao.

The authors are with the High Speed Electronics Laboratory, Department of Electrical Engineering, University of California, Los Angeles, CA 90095-1594 USA (e-mail: cchien@sst.com).

Digital Object Identifier 10.1109/TED.2005.850699
- Concurrent multiI/O's service for simultaneous and bidirectional communications on a shared transmission medium

- Realtime re-configurability in connectivity and bandwidth for optimized channel efficiency and fault-tolerance

Additionally, the fabrication of future interconnect systems must be compatible with the mainstream system-on-chip (SOC) and system-in-package (SIP) technologies for low-cost system production.

Traditional inter-chip and intra-chip communications are based solely on time-division multiple access (TDMA). In a TDMA-interconnect (TDMA-I) system, each I/O pair communicates over a shared transmission medium by transmitting only during its scheduled time slot in which no other I/O pair may transmit. In essence, time is being divided or allocated to each individual I/O pair so that a given transmission medium may be effectively shared. Furthermore, advanced TDMA-I (bus and links) in recent years has exploited multilevel signaling and dispersive signal equalization techniques to achieve multigigabits per second throughput [4]-[6]. Nevertheless, this type of system is limited to fixed and nonreconfigurable architecture that has high data transmission latency and can not support bidirectional and simultaneous transmission of multiple I/Os on the same physical channel.

To overcome the limitations of traditional TDMA-I, a number of new interconnect schemes have been investigated recently to greatly increase the aggregate data rate and concurrency as well as to reduce latency and power consumption [7]-[13]. These new schemes permit the use of a combination of other multiple access techniques, namely code division multiple access (CDMA) and frequency division multiple access (FDMA). In CDMA interconnect (CDMA-I), each I/O pair is assigned one or more pseudonoise $(\mathrm{PN})$ codes with near-ideal correlation property so that any other I/O pair assigned with different PN codes will contribute no interference when they are transmitting concurrently onto the same medium. In contrast, FDMA-I allows sharing of a transmission medium by assigning $\mathrm{I} / \mathrm{Os}$ to different frequency channels. I/Os assigned with different frequency channels may communicate concurrently with virtually no interference, provided that undesired frequency channels for a given I/O are filtered out properly. FDMA-I and CDMA-I may be combined into a multicarrier CDMA-I, whereby concurrent 
I/O transmissions are accomplished by properly assigning codes and frequencies to each I/O pair.

Fig. 1 compares new schemes based on CDMA-I and FDMA-I against the traditional TDMA-I in terms of two critical features for interconnects used in future ULSI systems: namely, aggregate data rate and re-configurability. Interconnect systems based on the multicarrier CDMA-I achieves the highest aggregate data rate and re-configurability. The high aggregate data rate is a result of the increased bandwidth made available through the use of more than one frequency channel. The high re-configurability arises from the increased number of combined code and frequency channels (or $N_{c}$ and $N_{f}$, which denote respectively the number of code channels and the number of frequency channels) now available to the system to dynamically assign based on the specific operation requirement. Note that both FDMA-I and CDMA-I have similar degrees of re-configurability but FDMA-I has higher aggregate data rates than CDMA-I due to the additional bandwidth made available through multiple frequency channels. In theory, TDMA-I has the same degree of re-configurability as either FDMA-I or CDMA-I in that a TDMA-I scheme can re-configure based on rescheduling and reassigning time slots for a given set of I/Os depending on the operation needs. In principle, the number of time slot $N_{t}$ can be made equal to $N_{c}$ or $N_{f}$. However, even though an increase in $N_{t}$ results in proportional reduction in the average data rate per I/O pin, the burst data rate for each $\mathrm{I} / \mathrm{O}$ pin still remains the same as the aggregate data rate. The high I/O bust data rate makes implementation difficult due to the need for high-order modulation and time-domain equalizers at high operating speed. For CDMA-I and FDMA-I, on the other hand, the burst data rate per I/O is inversely proportional to $N_{c}$ and $N_{f}$, which simplifies the signal processing required for each I/O. In particular, for CDMA-I, Rake receiver architecture may be employed to compensate for time dispersion in the transmission media but it requires much less complexity than a time-domain equalizer [14]. To put this into the perspective of practical implementation, wired TDMA-I has limited re-configurability due to difficulty in increasing $N_{t}$ without excessive complexity and power dissipation in the transceiver system design.

The re-configurability of wired TDMA-I may be improved with the single-carrier radio frequency (RF)-interconnect scheme (or SCRFI) shown in Fig. 1. The SCRFI uses only one frequency channel and achieves similar throughput as a wired TDMA-I system. However, it is able to achieve higher re-configurability than TDMA-I, since the transmission medium is no longer limited to fixed wiring but rather may be wirelessly broadcasted through coupling capacitors to communicate with different receivers. Such a scheme not only simplifies the fabrication process by eliminating the vertical metal studs needed in future three-dimensional (3-D) IC but also reduces the noise and dc power consumption. Transmission through capacitor-coupling can also be applied to FDMA-I, CDMA-I, and Multi-Carrier CDMA-I (or MCCDMA-I) as well.

In this paper, we will review recent progress in each of the new interconnect schemes described in Fig. 1 and discuss their applications in future ULSI interconnect implementations and architecture designs of next-generation computer/processor systems. We will first describe a wired CDMA-I that achieves

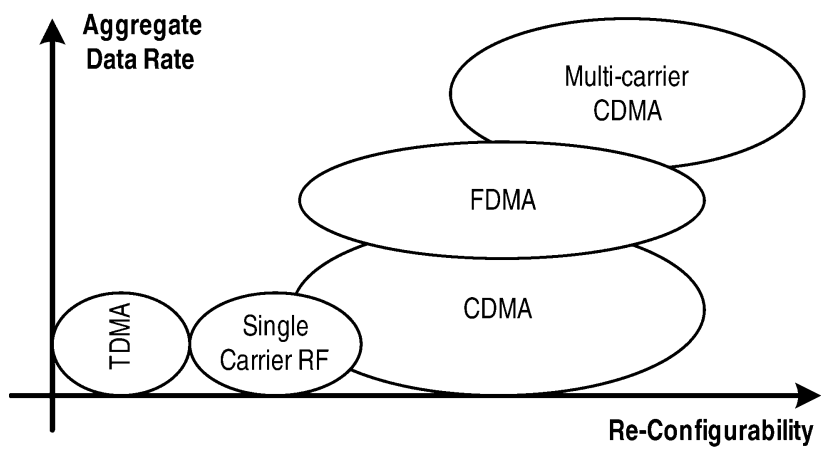

Fig. 1. Comparison of interconnect schemes.

channel re-configurability while providing simultaneous multiI/O's services. We will then discuss the wired FDMA-I that is able to achieve multiband (or multimode) channel communications. Subsequently, SC-RFI specifically designed for 3-D IC will be presented. Finally, wireless inter-chip communication based on MCCDMA-I will be discussed along with other system applications such as CDMA dynamic random access memory (CDMA-DRAM) and re-configurable interconnect for next-generation systems (RINGS).

\section{CDMA-INTERCONNECT}

Conventional TDMA-I bus and links are designed for nonreconfigurable system architectures and have extensive data bus latency and limited system performance. Therefore there is a great demand for improving the efficiency of both on-chip and off-chip interface circuits without increasing the cost and overhead. This could be achieved by bus-oriented and system-oriented approaches by changing the nature of the request stream, increasing the channel concurrency and decreasing the channel latency. To accomplish this, we have proposed using the CDMA-I for future re-configurable SOC [10].

The proposed CDMA-I uses different PN codes to separate different users and I/O ports on a shared bus. The CDMA-I modulates the data into spread spectrum signal by orthogonal Walsh or PN codes [14]. For $N$ I/O ports, the minimum spread ratio is $\log _{2} N+1$. The code-modulated signal from each I/O is combined into a multilevel signal on the channel. Re-configurability is a unique advantage of the CDMA-I because the receiver can detect data sequence of different I/Os by simply changing codes through firmware rather than additional hardware needed for retiming and/or framing of different users. Because the same code can be used in different CDMA demodulator, the CDMA-I transceiver is more than an $N$-to- $N$ data switch but rather could implement any $N$-to- $M(M \leq N)$ I/O mapping. Moreover, the CDMA-I allows not only re-configurability in connectivity but also in bandwidth, whereby one or more I/Os could be allocated more bandwidth by simply assigning more than one code to the specific I/O. It can also reduce the communication overhead in a packet switched system by eliminating the need for having I/O port addresses in the headers of the transmitted packets. The CDMA-I can be used for re-configurable chip interconnects [10], wired busses [11] and future gigascale integrated systems. Fig. 2(a) shows a 4-port system interconnected by the 


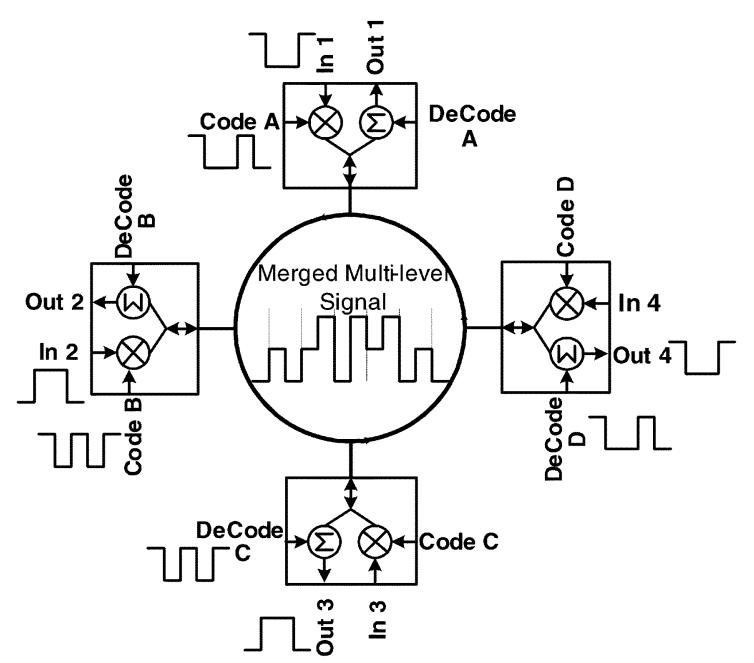

(a)

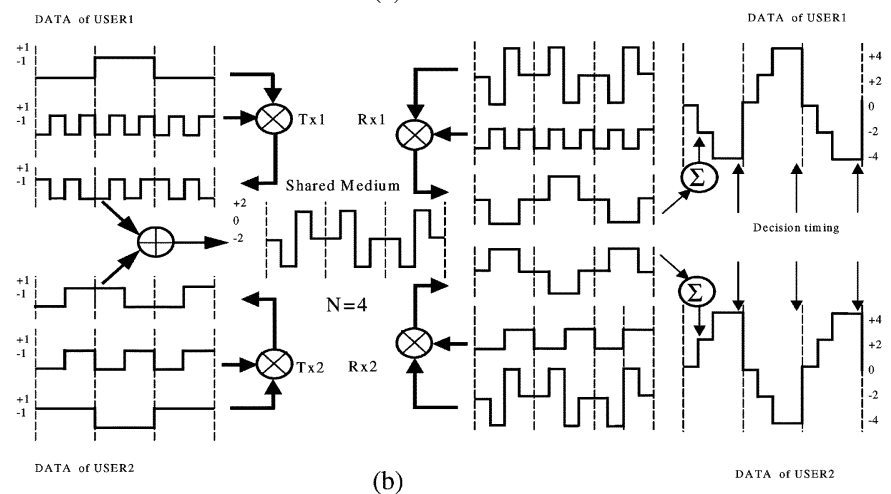

Fig. 2. (a) A 4-port system implemented by the CDMA-I transceive. (b) Modulation of data and spreading for two simultaneous I/O users.

CDMA-I. Each port on the bus sends a coded data, which can be recovered by any other port with the corresponding code key. Fig. 2(b) gives an example of spreading, de-spreading and correlating DATA by using orthogonal Walsh codes in a CDMA-I with two concurrent I/O users.

Fig. 3 shows the CDMA-I transceiver architecture, which implements the transceiver for each of the port shown in Fig. 2(a). The transmitter consists of a baseband CDMA-modulator to modulate the user data with the assigned code, a Walsh code generator to provide the orthogonal data "keys", a data combiner to put together all the user data into a serial data sequence with multiple signal levels for transmission, a phase-lock-loop clock generator, and finally a 50-ohm matching output buffer to transmit the signal. The receiver picks up the multilevel signal transmitted through an inter-chip interconnect line; similar input matching network at the transmitter is used. As the first step, the clock is recovered from the received multilevel signals, and the signal is quantized into a multibit data. The I/O's data can be recovered by demodulating the multibit data with corresponding Walsh codes. An asynchronous error-detection correlator is designed to obtain synchronization of the input data within one-symbol clock period.

Since the received signal has multiple values, a multilevel signal clock-data-recovery (CDR) circuit is needed to synchronize the clock and recover the baseband multilevel data. To fully utilize the edge information of the input multilevel signal

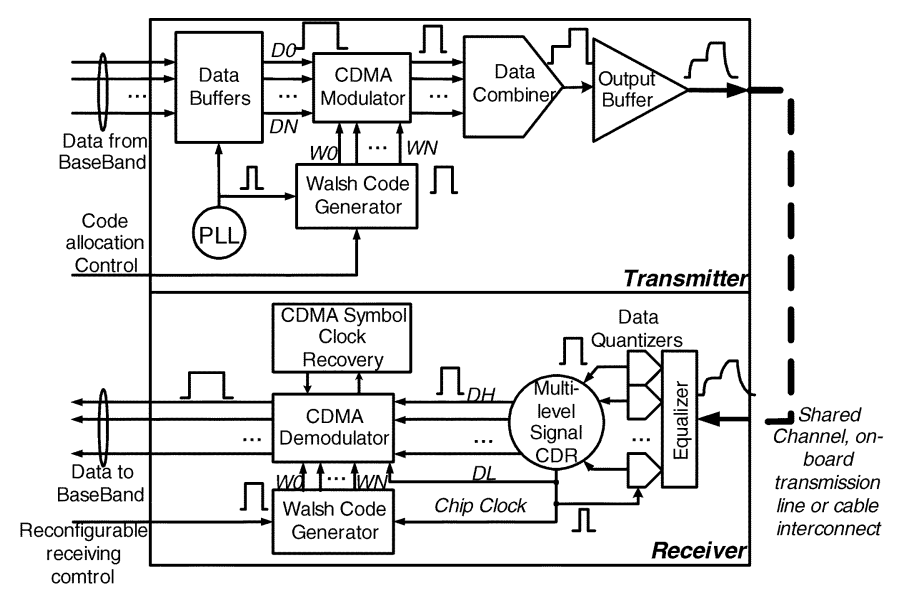

Fig. 3. CDMA-I interconnect transceiver architecture.

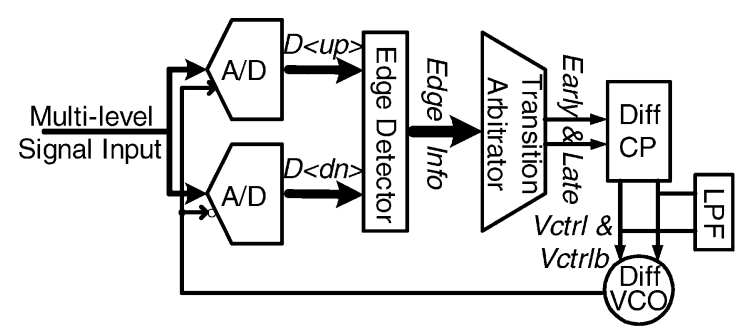

(a)

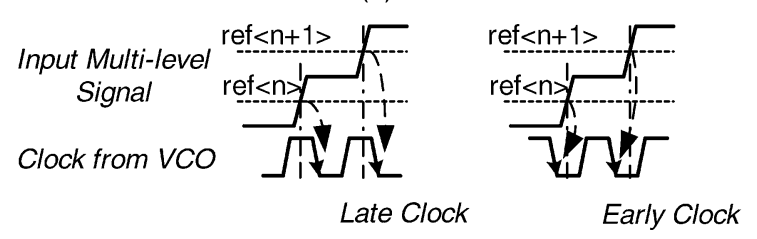

(b)

Fig. 4. (a) Multilevel signal clock data recovery circuit. (b) Early and late clock decision.

and avoid extra coding to decrease the transfer efficiency [15], a multilevel clock data recovery circuit with Alexander-type phase detector is designed that uses all data transitions to reduce jitter in the recovered clock and data. This turns out to be possible because the received data has more data transitions due to modulation by different Walsh codes. Fig. 4(a) shows the Alexander-type clock data recovery circuit architecture for the multilevel input signal. It consists of two A/D converters, which are used to quantize the input signal at the rising and falling edge of the clock. To achieve immunity to noise on the control line so as to satisfy the jitter requirement of the CDMA-I while using less silicon area, a differential control VCO using symmetrical MIS varactor pairs is implemented [10].

A $2 \times 2$ re-configurable $C D M A-I$ transceiver has been implemented in TSMC $0.18 \mu \mathrm{m}$ CMOS technology. The entire interconnect system has been implemented with the transceiver chip-set packaged in TQFP on a printed-circuit board with 31-mil thick FR4. Fig. 5(a) shows the two transmission data sequence D0 and D1. Both of them are modulated by different Walsh codes and combined together into multilevel signal outputs, whose peak-to-peak voltage is $400 \mathrm{mV}$. Fig. 5(b) shows the received data eye of the multilevel signal output. Fig. 6(a) shows the recovered clock spectrum at $2.65 \mathrm{GHz}$ and 


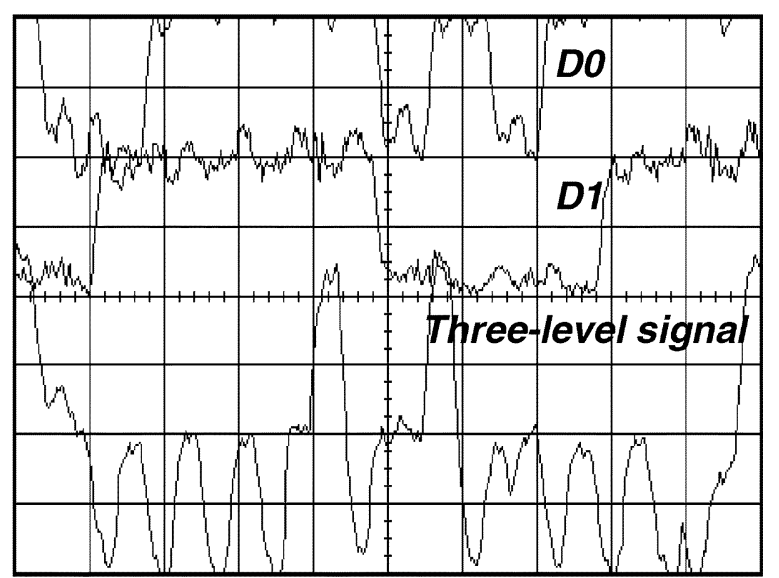

(a)

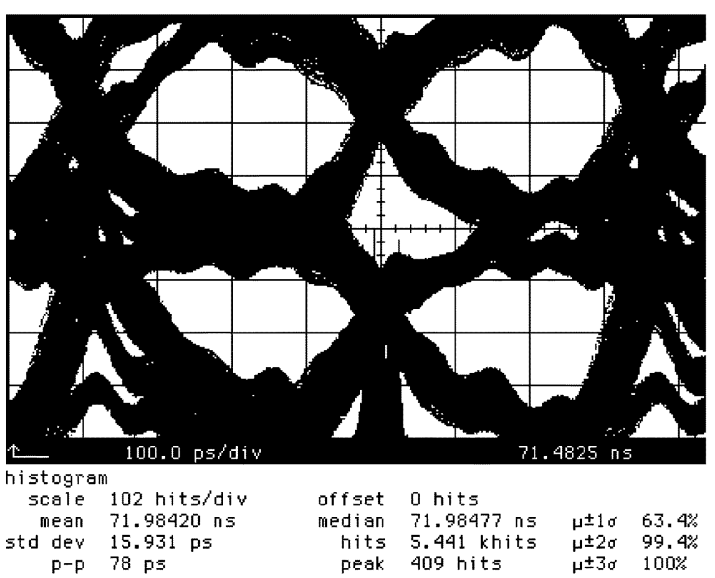

(b)

Fig. 5. (a) Transmitter data sequences, three-level signal output. (b) Received data eye through a $24-\mathrm{cm}$ cable (1.5-GHz bandwidth).

(a)

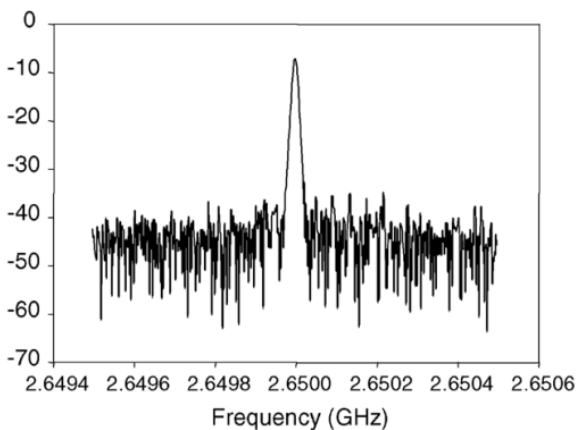

(b)

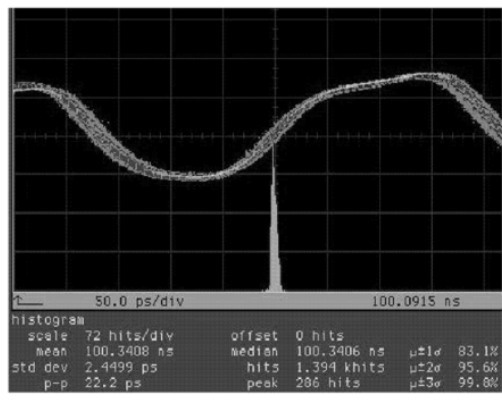

Channel Reconfigure

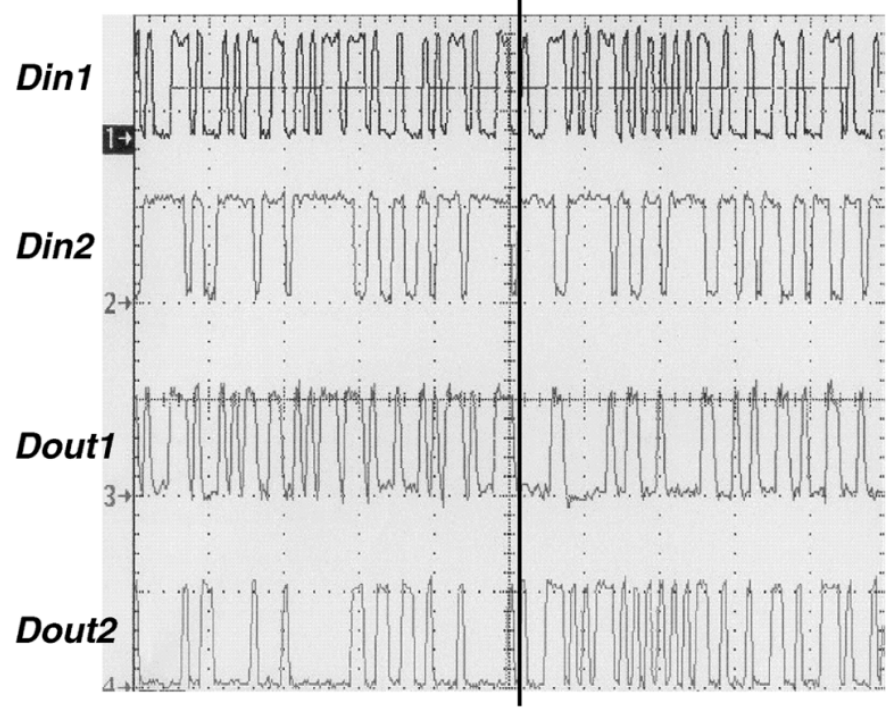

(c)

Fig. 6. (a) Recovered clock spectrum. (b) Recovered clock histogram, and jitter. (c) Waveforms of CDMA transmitter and receiver.
Fig. 6(b) shows the histogram of the recovered clock. When the input is a $2^{23}-1$ pseudorandom data sequence, the measured root-mean-square (rms) jitter of the recovered clock is less than 2.5 ps. Fig. 6(c) shows the waveform of the input data of the CDMA transmitter and the output data of the CDMA receiver. As shown, the "on-the-fly" re-configurability has been realized. The total area of the $2 \times 2$ transmitter and receiver is $0.6 \mathrm{~mm}^{2}$. With $1.8 \mathrm{~V}$ as the power supply voltage, the transmitter draws $37 \mathrm{~mA}$ and the receiver draws $45 \mathrm{~mA}$. The transmitter and receiver of each I/O pair totally consume $74 \mathrm{~mW}$.

\section{FDMA-INTERCONNECTS}

Fig. 7(a) and (b) show conventional TDMA-I links that transmit digital data either directly or in a PAM form [16]. Those signals occupy only the lowest frequency band (i.e., the baseband) of the physical channel. One may envision the possibility of transmitting data concurrently in RF-modulated frequency bands to extend the data rate of the same physical channel. With proper frequency band separations, transmitted RF-modulated signals can be allocated in spectrum to minimize the cross-band interference among all available bands. With that in mind, we have developed a novel RF/baseband combined FDMA-Interconnect (FDMA-I) scheme, shown in Fig. 7(c), to allow additional data bandwidth over the conventional baseband-only interconnect. We also find that the FDMA-I data transmission can be designed to support the bidirectional communication link without extra overhead.

FDMA-I can also be adopted to improve the interface bus performance. Fig. 8(a) shows a conventional parallel bus which suffers from interference from adjacent wires, which becomes more serious as the spaces become more compact [17]. This problem can be largely eliminated when transmitting data on adjacent wires in different RF-modulated frequencies as shown in Fig. 8(c), [9]. Fig. 9 shows the advantage of a FDMA-I system in reducing the cross-band interference. According to system simulations, more than $20-\mathrm{dB}$ attenuation may be obtainable when transmitting $2+2$ Gbps simultaneously via adjacent RF and basebands, separated by the carrier frequency of $8.5 \mathrm{GHz}$ without extra signal filtering. Fig. 10 shows a dual-band example of a system based on FDMA-I. The physical transmission line is terminated at its characteristic impedance in both 


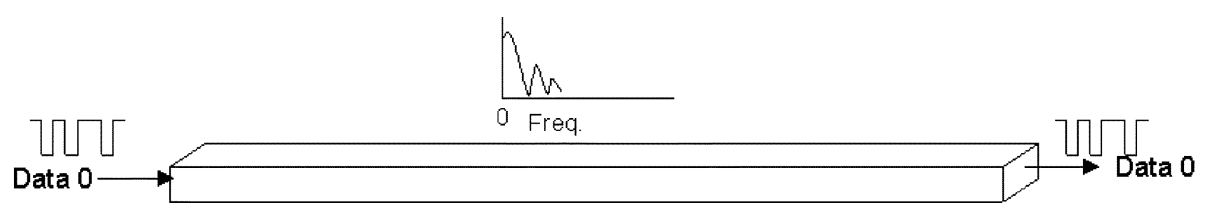

(a)

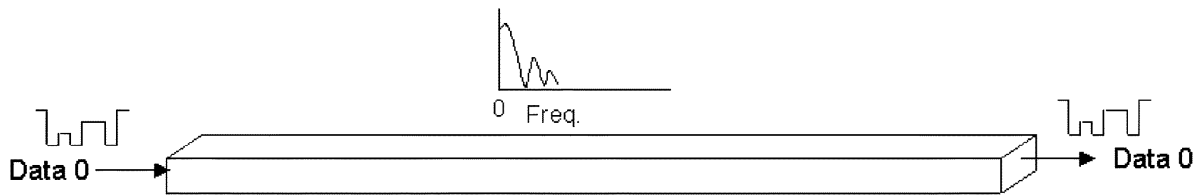

(b)

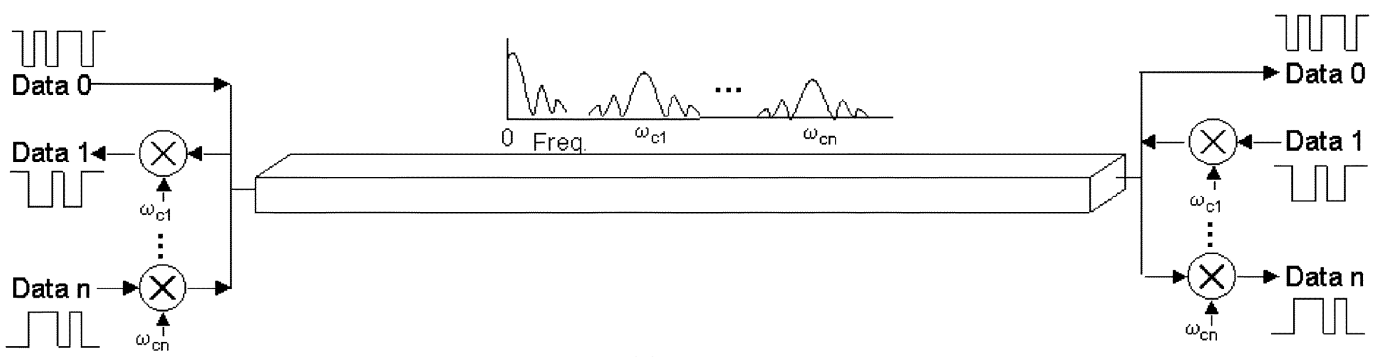

(c)

Fig. 7. (a) Conventional direct digital interconnect. (b) PAM signal in conventional digital interconnect. (c) FDMA-Interconnect (FDMA-I).

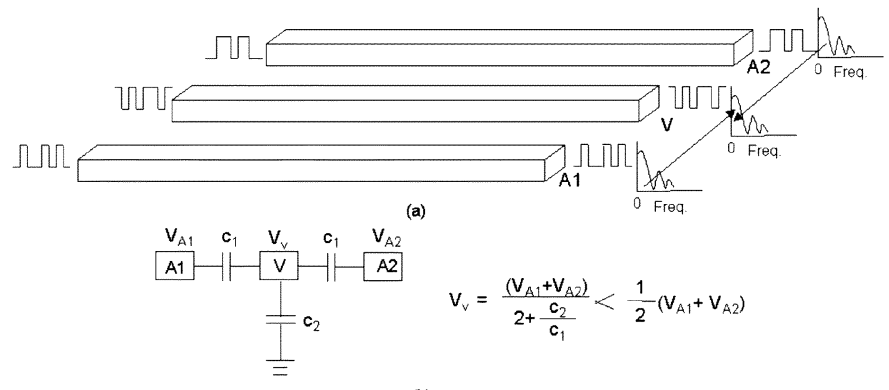

(b)

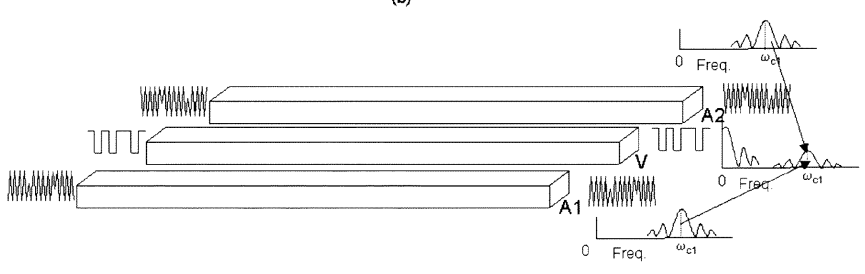

(c)

Fig. 8. (a) Conventional parallel bus interconnects. (b) Simplified circuit model for capacitor coupling of adjacent wires. (c) Parallel FDMA-I bus with RF-modulated aggressors.

ends to eliminate signal reflections. Each I/O port contains baseband and RF-band transceivers to support simultaneous multiband data communication. The above FDMA-I can be reconfigured to route between $N \times N$ I/Os by selectively switching among various frequency bands and also possibly supporting both uni-directional and bidirectional data transmissions.

The FDMA-I transceiver prototype chip has been implemented in $0.18 \mu \mathrm{m}$ CMOS [18]. The test set-up consists of an inter-chip interconnection situated on a FR4 board with a $10-\mathrm{cm}$ 50-ohm micro-strip line and two prototype chips wire-bonded to the ends of the line. Measurements were taken at a total data rate of 4 (or $2+2$ Gbps in RF and baseband, respectively) Gbps/wire in both uni-directional and bidirectional modes. Eye diagrams of received data under bidirectional transmission are

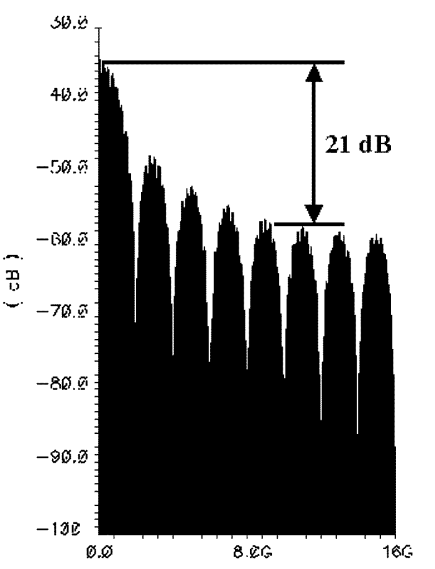

2Gbps Base Band data in spectrum

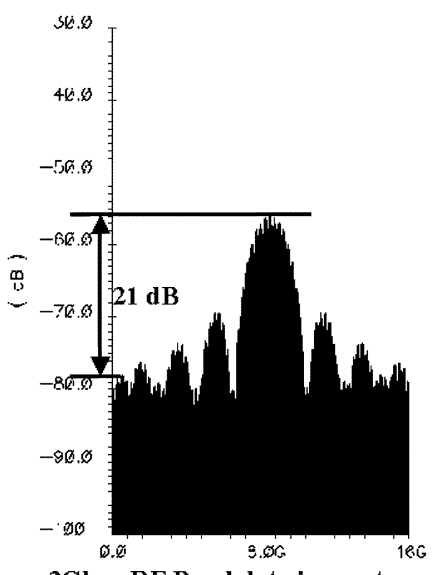

2Gbps RF Band data in spectrum

Fig. 9. More than $20 \mathrm{~dB}$ signal attenuation obtained for $2+2$ Gbps data rate transmissions via RF and the baseband, separated by an $8.5-\mathrm{GHz}$ carrier.

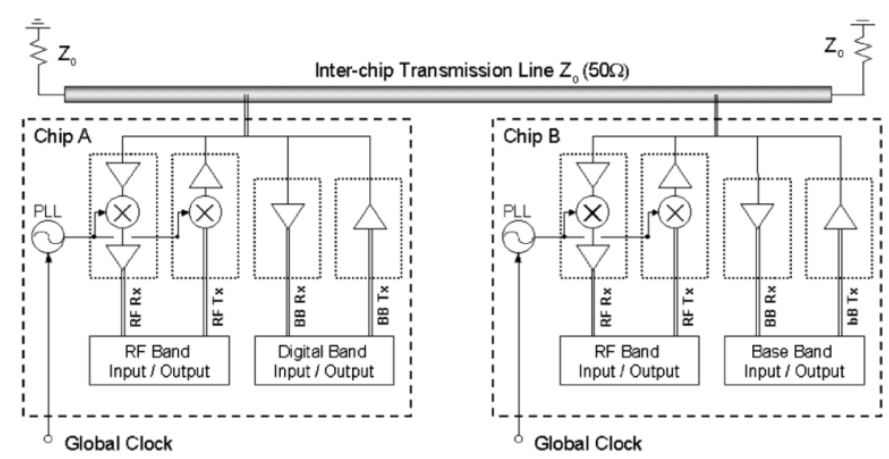

Fig. 10. Fual-band FDMA-I system.

shown in Fig. 11(a). R.M.S. jitters of the received data, shown in Fig. 11(b)-(c), are 19.4 and 34.6ps for the baseband and RF-band, respectively. Transmitted and received data patterns of the baseband and RF-band (inverted data) are compared in Fig. 11(d). 


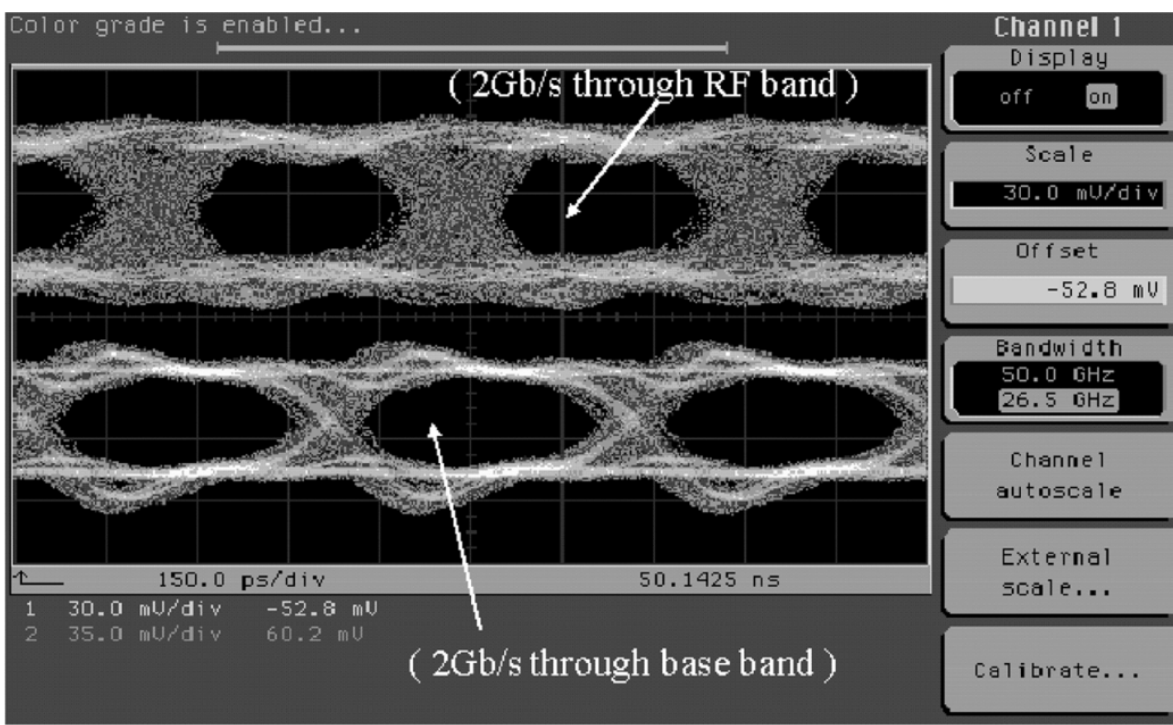

(a)

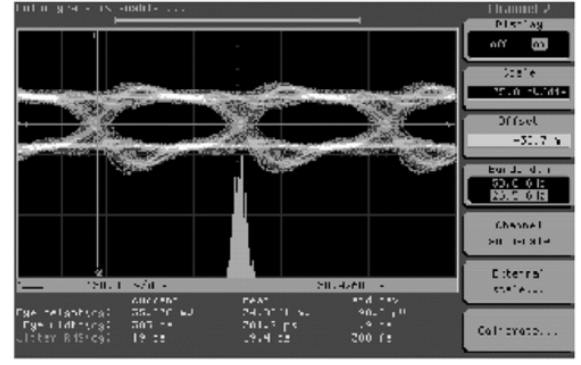

(b)

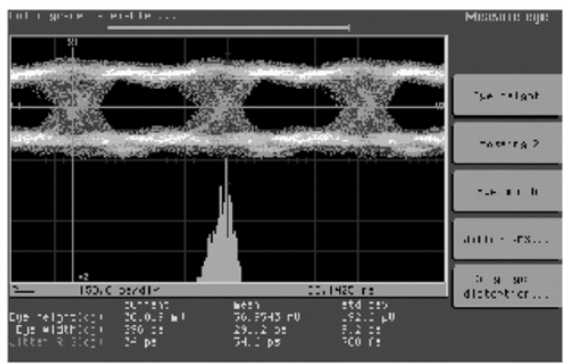

(c)

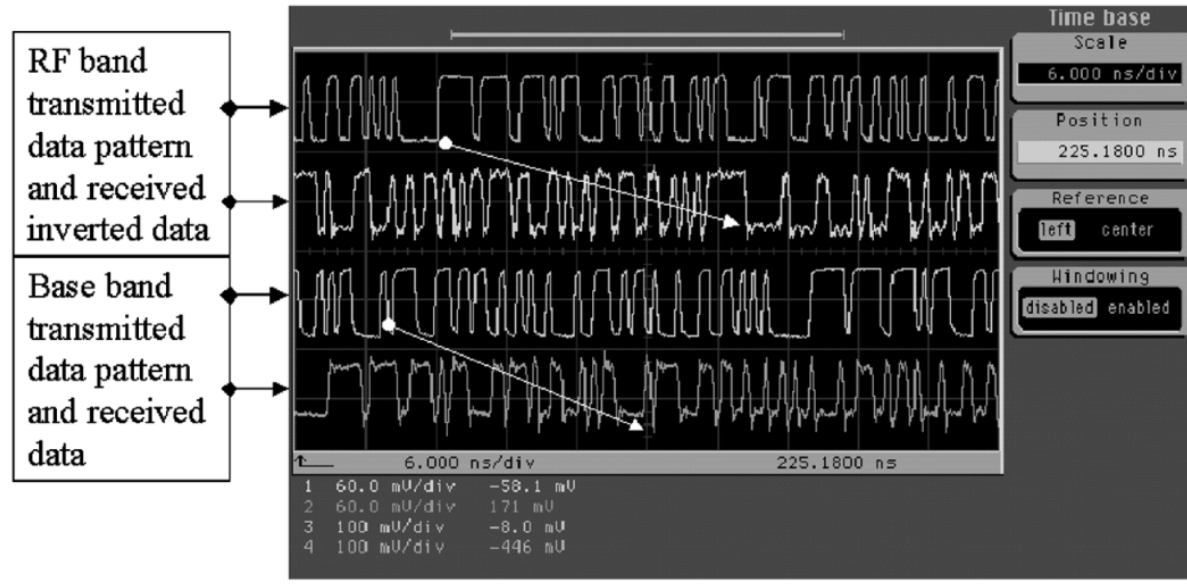

(d)

Fig. 11. Measured eye diagrams and waveforms of the dual-band bidirection FDMA-I data transmission at $2+2 \mathrm{~Gb} / \mathrm{s} /$ wire data rate. (a) Received data eye diagram in $4 \mathrm{~Gb} / \mathrm{s}$ bidirectional data transmission. (b) Received base band RMS data jitter $=19.4$ ps. (c) Received RF band RMS data jitter $=34.6$ ps. (d) Transmitted pattern and Received waveform in $4 \mathrm{Gbp} / \mathrm{s} /$ wire (bidirection).

These results demonstrate that the FDMA-I system can provide high data rate and bidirectional communications with sufficient jitter performance. The jitter performance can be further improved by using more sophisticated signal filtering and impedance matching techniques.

\section{Single CARRIER RF-INTERCONNECT FOR 3-D IC}

With the dramatic developments in semiconductor technology and circuit designs, more sophisticated systems have been implemented on a single chip. While the expanding market keeps pushing for the higher speed, lower power, more powerful and less costly single chip systems, it is actually becoming more difficult to use conventional planar technology to design multifunction and low-cost chip systems especially in deep submicron technologies due to high parasitic capacitance, short-channel effect and strong crosstalk between interconnect [19]. Furthermore, conventional planar technology also faces fundamental physical limits and will encounter more significant interconnect issues in the future. All of these issues heavily 


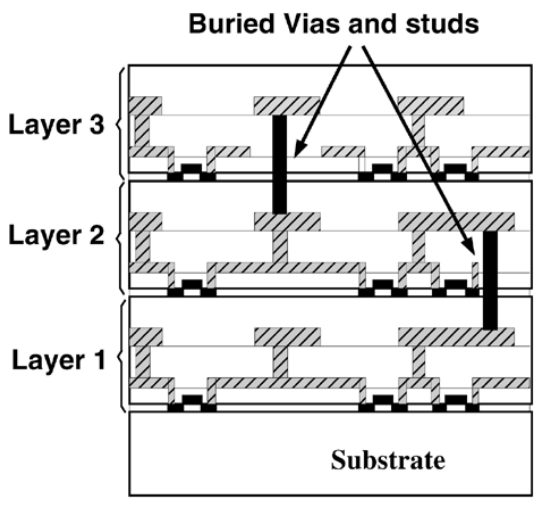

Fig. 12. Buried via/stud interconnect method.

impact the next-generation IC development. A 3-D IC (3D IC) has been proposed to overcome the above drawbacks to allow stacking of active device layers or chips. With this alternative, 3-D IC will surpass traditional two-dimensional (2-D) IC in reduction of chip area, power consumption, timing constraints and even cost [20]. Therefore, 3-D IC has gradually become a mainstream in future ULSI development.

In 3-D IC, several key obstacles must be surpassed, one of which is to connect multiple device layers effectively. Traditionally, vertical interconnects are formed by etching via through layers and depositing metal studs to physically connect various active device layers (Fig. 12) [21]. This manufacturing method, however, becomes less manufacturable when the total number of vertical active layers becomes large, leading to increased etching depth and vertical line parasitics. In order to overcome the above drawbacks, a self-synchronized single carrier RF-Interconnect (SCRFI) has been proposed [13]. Unlike the wired via/stud interconnects, the proposed SCRFI is based on wireless capacitor-coupling and peak-signal detection. Since the coupling is accomplished through capacitors, there is no need of fabricating via and stud and would consume no dcpower along the transmission media.

The $S C R F I$ is also different from the previously discussed RF-Interconnect (RFI). The RFI reported previously in [9], [18] requires the transmitter to up-convert the baseband signal using an RF carrier and the receiver to down-convert the signal with the same frequency RF carrier for data recovery. Although it enables effective data transmission for inter-chip applications, the RFI is less efficient for extremely short distance inter-layer communication applications in 3-D IC. First, the RFI needs precise local oscillators (LOs) in both transmitter and receiver for signal modulation and demodulation, which increase the design complexity and manufacturing cost. Second, the LOs at both sides must be synchronized, which require matching crystal and power hungry oscillators and phase locking circuits.

To surpass the above drawbacks, a SCRFI with smaller chip area and less power dissipation has been realized by using a simple signal peak-detector in the receiver for baseband signal recovery [13]. This self-synchronized (or noncoherent) interconnect scheme does not need LO for frequency demodulation in the receiver and extra phase-locked-loop circuit for data synchronization.

This self-synchronized SCRFI circuit architecture is shown in Fig. 13, in which the transmitter (Tx) includes an input buffer

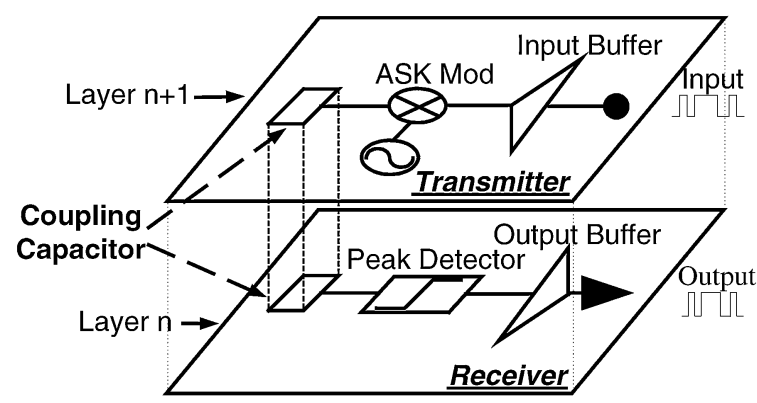

Fig. 13. SCRFI circuit architecture.

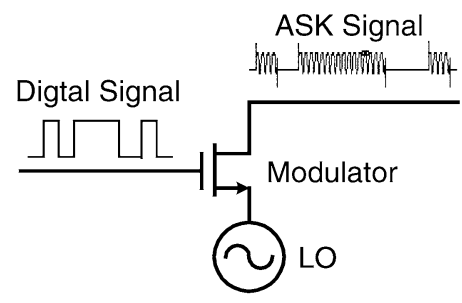

Fig. 14. ASK modulation.

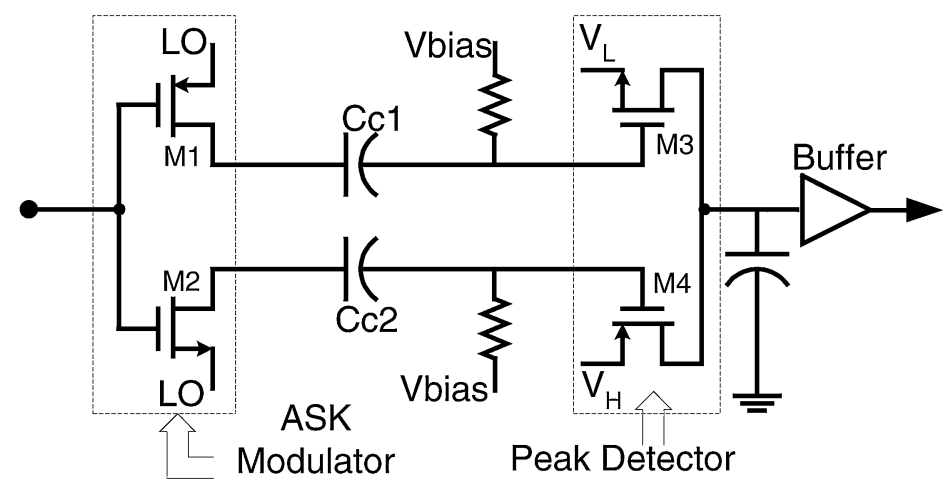

Fig. 15. Peak signal detection CMOS circuit.

circuit and an ASK modulator [22]; the receiver (Rx) consists of a signal peak-detector and an output buffer. Due to the high pass nature of the inter-layer capacitor coupling (Fig. 13), the baseband signal is first up-converted by the LO carrier. ASK modulation is chosen for its simplicity and efficiency. The ASK signal can be generated by switching on and off the LO carrier through a modulator, as shown in Fig. 14. When the baseband signal is high, the LO carrier is switched on, otherwise the LO is blocked. The ASK modulated signal, after passing through the channel, is recovered in the Rx by the peak-detector, in which both pMOS and nMOS are used to be as diodes so that logic "1" and logic " 0 " can be equally and effectively passed without the threshold loss, as shown in Fig. 15. An output buffer following the peak detector is used to rectify the signals and drives the off-chip load.

A prototype SSRFI has been implemented in $0.18-\mu \mathrm{m}$ CMOS technology [13]. Fig. 16 demonstrates the eye diagram of the received data from a 3-Gbps PRBS input data stream modulated by a $10-\mathrm{GHz} \mathrm{LO}$. The eye height and width are about $220-\mathrm{mv}$ rms and 257-ps rms, respectively. Fig. 17 shows an excellent output signal jitter of $1.28 \mathrm{ps}$. The measured bit error rate (BER) is below $1.2 \times 10^{-10}$. The coupling capacitance is chosen as 60 


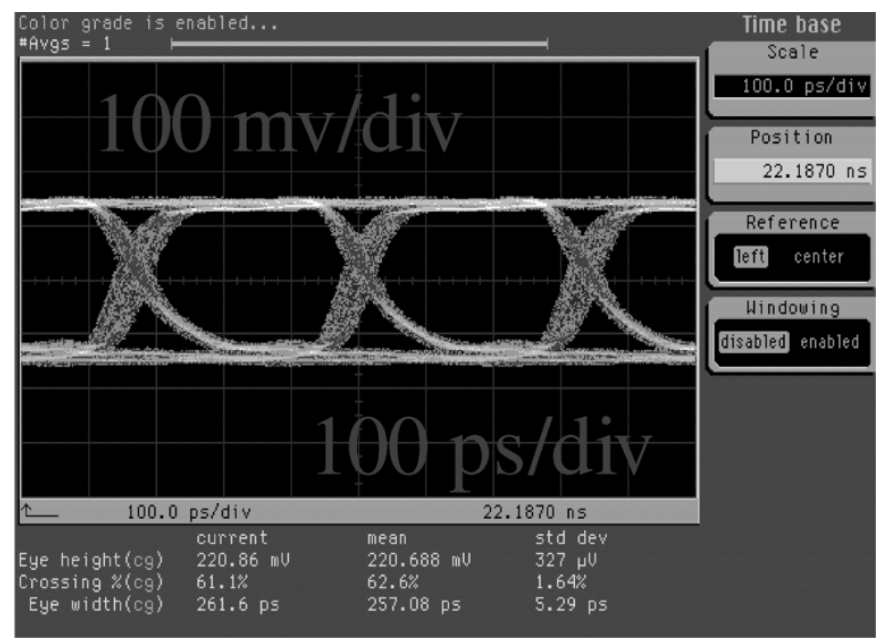

Fig. 16. Eye diagram of output signal at $3 \mathrm{~Gb} / \mathrm{s}$.

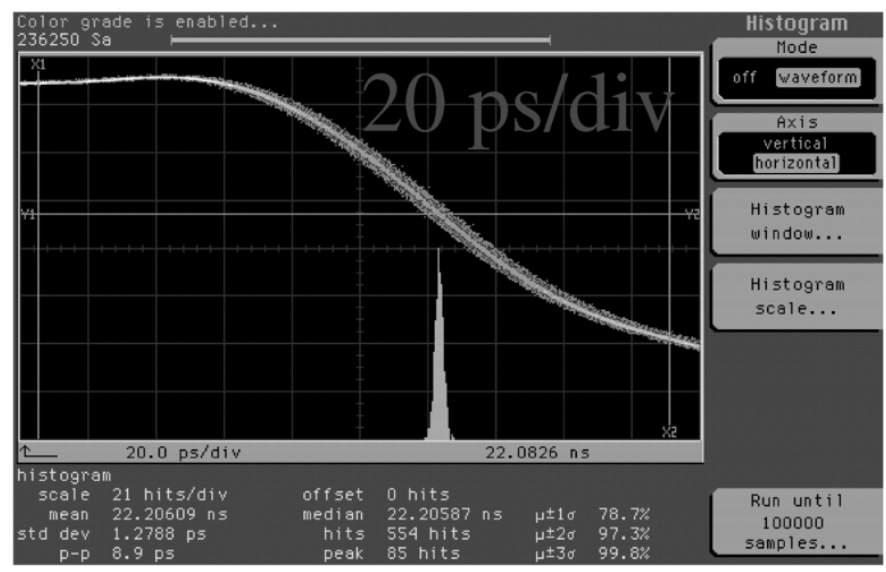

Fig. 17. Jitter measured at $3 \mathrm{~Gb} / \mathrm{s}$ PRBS.

$\mathrm{fF}$ in this particular demonstration. Theoretically it can be as low as $10-20 \mathrm{fF}$ when assuming the bias resistance is set at $10-20$ $\mathrm{K} \Omega$ to ensure the high-pass corner frequency being significantly lower than that of LO. Since the required coupling capacitance is rather low, the implementation of such capacitors should be straightforward in future 3-D ICs.

\section{System APPlications of New INTERCONNECT TECHNOLOGIES}

New generations of SOC or SIP require all the previously mentioned properties of interconnects with high throughput, low latency, and high bandwidths. Deep-submicron and nano technologies add numerous extra challenges to these requirements, such as wire delays, reliability issues, jitter effects, and so on. However, system and application requirements add two additional challenges to this list: low power (and low energy) and re-configurability.

In this section we will discuss the requirements of these nextgeneration systems. We will illustrate advantages and needs for new types of interconnect to address these issues. Three types of systems will be depicted: a general purpose compute platform, a wireless multi-carrier CDMA-interconnect (MCCDMA-I) for SIP integration and the RINGS platform. (a)

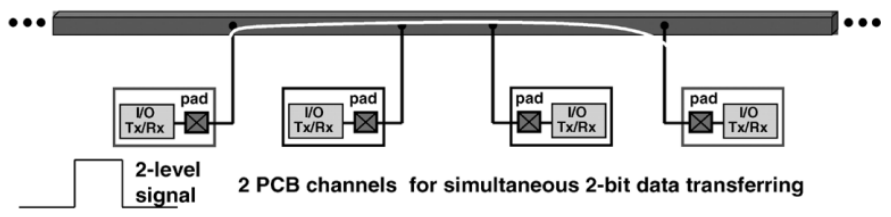

(b)

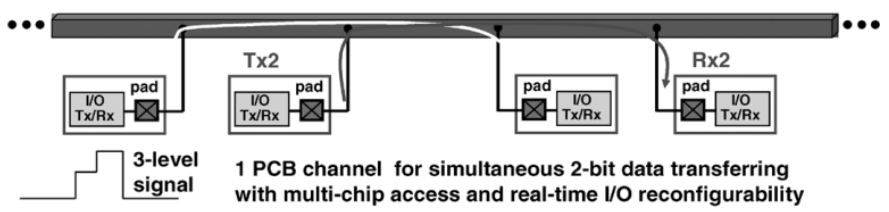

Fig. 18. (a) Conventional bus. (b) Source synchronous CDMA-I bus.

\section{A. General Purpose Computing Platform}

The communication between a CPU and the main memory system are performance-limited by the behavior of the bus. Conventional TDMA-I based memory busses are designed for fixed and nonreconfigurable systems and have extensive data bus latency. Therefore, there is a great demand for improving the efficiency of the processor-to-main memory interface without increasing the cost and overhead.

The bus interface based on CDMA-I described before in Section II can be used to achieve several goals: reduced latency, increased bandwidth, re-configurability and reduced energy consumption. It achieves this by utilizing both $C D M A-I$ and a variation of source synchronous clocking for multilevel superposition. The multiple off-chip transmitters can occupy the same channel at the same time, but are separated from each other by using a set of orthogonal code [14]. This source synchronous CDMA interconnect (SSCDMA-I) bus interface, which can be applied for small memory subsystems and chip-to-chip interconnects including processor-to-cash bus, back-side bus, and shared memory multiprocessor systems, improves the system performance due to the increased channel concurrency and decreased data bus latency. Lower power and more cost-effective system is also achieved owing to the use of reduced number of pins and PCB traces and smaller die and package size [11], [12].

The cost associated with DRAM memories increases with the number of I/O pins on the DRAM package. The performance of a memory system could be increased somewhat by widening memory channels and/or providing independent DRAM banks. However, both these approaches increase the cost; furthermore, channel latency and concurrency problems still exist. High-speed narrow channels used Direct Rambus DRAM (D-RDRAM) may suffer from long channel latency: for instance, if two read requests arrive at DRAM1 back-to-back or two read requests arrive at DRAM1 and DRAM2, respectively, the second request must stall until the first request finishes using the shared data bus. Although increasing the bus speed improves the performance somewhat, channel request latency still exists.

The conventional bus interface, as shown in Fig. 18(a), uses binary signaling and requires two PCB channels for 2-bit data transferring simultaneously. Only one transmitter and one receiver can access the shared bus simultaneously; the other devices should wait until the two devices finish their job. Thus this 


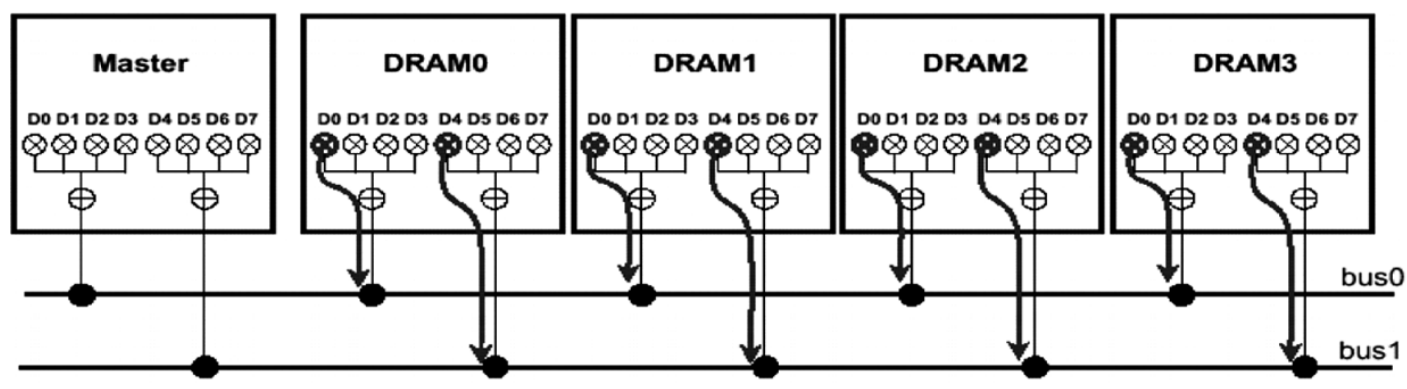

(a)

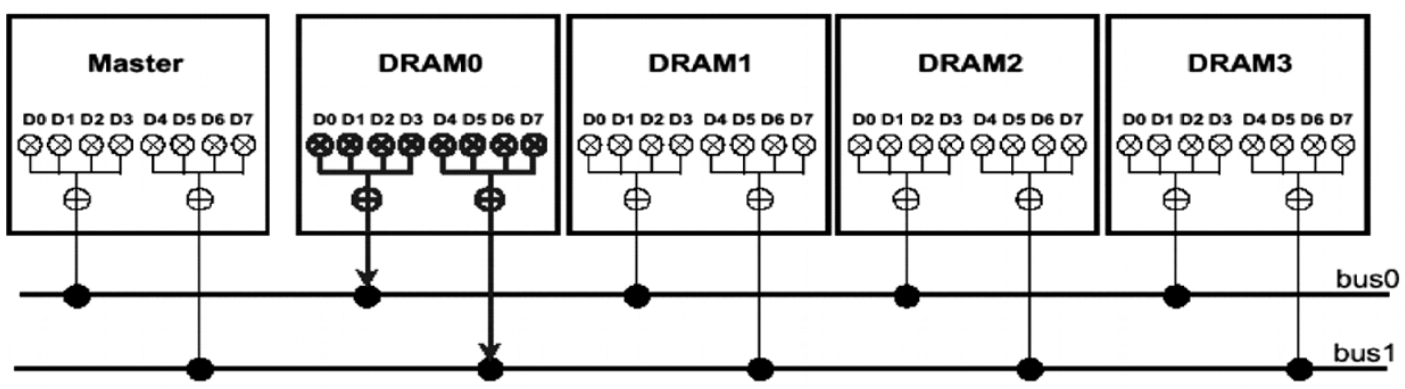

(b)

Fig. 19. (a) Reconfigurable CDMA DRAM: The memory control reads the first bit of each DRAM on bus 0 and the fourth bit on bus 1 . (b) Re-configurable CDMA DRAM: The memory controller reads all 8 bits from DRAM 0 .

\begin{tabular}{|c|c|c|c|c|c|c|c|}
\hline & $\begin{array}{c}\text { \# of } \\
\text { high-speed } \\
\text { Data } \\
\text { Channels }\end{array}$ & $\begin{array}{l}\text { \# of Parallel } \\
\text { High-speed } \\
\text { Address } \\
\text { Channels }\end{array}$ & $\begin{array}{c}\text { \# of Parallel } \\
\text { High-speed } \\
\text { Clock } \\
\text { Channels }\end{array}$ & $\begin{array}{l}\text { Total } \\
\text { \# of High- } \\
\text { speed } \\
\text { Data \& Add. } \\
\text { Channels }\end{array}$ & $\begin{array}{l}\text { Min. \# of } \\
\text { Shielding } \\
\text { Channels } \\
\text { for } \\
\text { Data \& Add. }\end{array}$ & $\begin{array}{l}\text { Data Rate } \\
\text { (Mbps) }\end{array}$ & $\begin{array}{c}\text { Total } \\
\text { Data } \\
\text { Bandwidth }\end{array}$ \\
\hline SDRAM (PC133) & 64 & 12 & 1 & 76 & - & 133 & 1.1 Gbyte/s \\
\hline D-RDRAM & 16 & 8 & $\stackrel{2}{2}$ (differential) & 24 & 24 & $400 * 2$ & 1.6 Gbyte/s \\
\hline DDR(SSTL-2) & 64 & 12 & $\begin{array}{c}8 \\
\text { (data strobe) }\end{array}$ & 76 & - & $133 * 2$ & 2.1 Gbyte/s \\
\hline CDMA DRAM & 8 & 4 & $\begin{array}{c}2 \\
\text { (differential) }\end{array}$ & 12 & 12 & $800 * 2$ & 1.6 Gbyte/s \\
\hline
\end{tabular}

Fig. 20. Comparison of memory buses.

end-to-end request delay increases the channel latency. However, in the SSCDMA-I Bus interface, as shown in Fig. 18(b), two off-chip transmitters and two receivers can access the shared bus simultaneously using only 1 PCB channel for 2-bit data transferring, thus the end-to-end data request delay is removed and the channel latency is decreased. And, (for the same data rate) the required number of channels becomes half. This reduced number of channels decreases the channel power consumption and the overall system cost.

The application of SSCDMA-I to a DRAM memory subsystem is illustrated in Fig. 19. It shows an example with two busses, each having a 4-to-4 CDMA DRAM interface. In the first case, the SSCDMA-I has been configured to read the first bit of each DRAM on bus0 and the fourth bit on bus1. By a simple re-configuration, the memory controller reads all 8 bits of DRAM0.

This is one illustration of the reconfiguration-on-the-fly: it shows how the memory bandwidth and memory architecture can be adapted to the application running on the CPU. For instance, streaming video has a different memory transfer pattern than data packet routing or desktop applications. Thus we envision that in the future the software or the operating system, can adjust the memory and cache hierarchy to bring the data being processed "closer" to the CPU and in this way, bandwidth, and performance are improved while the energy consumption remains low.

Fig. 20 shows the brief characteristics comparison of the CDMA DRAM system and the other conventional DRAM systems. Since the SSCDMA-I bus interface uses the current mode output drive the power dissipation in a terminated bus is only a function of the total number of high-speed buses [11]. This proposed re-configurable DRAM uses half the number of high-speed buses of the conventional D-RDRAM and hence reduces the channel power dissipation by at least 50\% [11], [12]. 


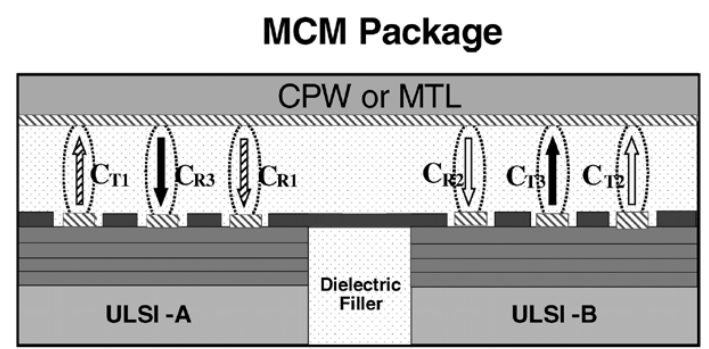

Top View

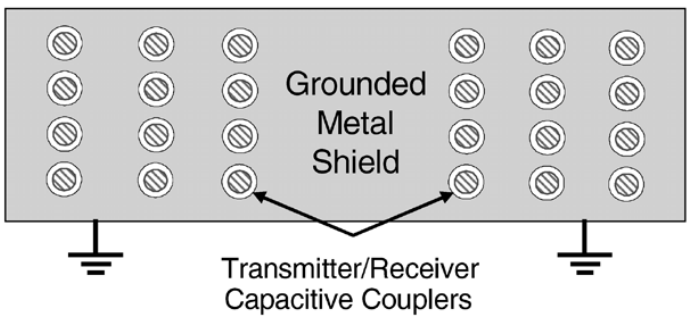

Fig. 21. MCCDMA-I system-miniature LAN inside a MCM package.

\section{B. Wireless MultiCarrier CDMA-Interconnect (MCCDMA-I) for SIP}

In Section IV, we have described the use of a single carrier RF-interconnect (SCRFI) for vertical integration of 3-D ICs through interlayer capacitor coupling. In that case, the communication distance is extremely short (on the order of $10 \mu \mathrm{m}$ ). A simple ASK-modulation used in the transmitter and a self-synchronized peak detection used in the receiver are proven to be sufficient for achieving effective interlayer data communication. The situation however is different in forming an efficient interconnect system for longer distance and multichannel communications inside the SIP. The future SIP interconnect systems would require wide bandwidth, short latency, real-time re-configurability and simultaneous multiI/O's service. To meet such system needs, we have developed a MCCDMA-I (multicarrier CDMA-Interconnect) by combining the FDMA-I and CDMA-I to take advantages from both systems [7], [9]. The $M C C D M A-I$ can be connected in either wired (via direct-coupling) or wireless (via capacitor-coupling) fashion depending on specific system applications. The possibility of using the MCCDMA-I in wireless manner is discussed as follow, which has advantages of eliminating the dc power consumption of the transmission line and the baseband switch noise due to the high pass nature of the capacitor coupling.

The intended MCCDMA-I wireless interconnect system is illustrated in Fig. 21 as a miniature wireless local area network (LAN) located inside a SIP (or a MCM) [7]. Like any other wireless communication system, this miniature LAN contains ULSI I/Os as users, capacitor couplers as near field antennas, RF transceivers and off-chip but in-package MTL (microwave transmission line) as a shared broadcasting medium. Output signals can be up-linked to MTL via transmission capacitive couplers $\left(\mathrm{C}_{\mathrm{T}}\right)$, then down-linked via receiving capacitive couplers $\left(\mathrm{C}_{\mathrm{R}}\right)$ to input ports to fulfill the interconnect function. Electrodes of capacitor couplers can be easily formed between the uppermost ULSI metal and the MTL. With a shared MTL, combined FDMA/CDMA multiple-access techniques can be used to

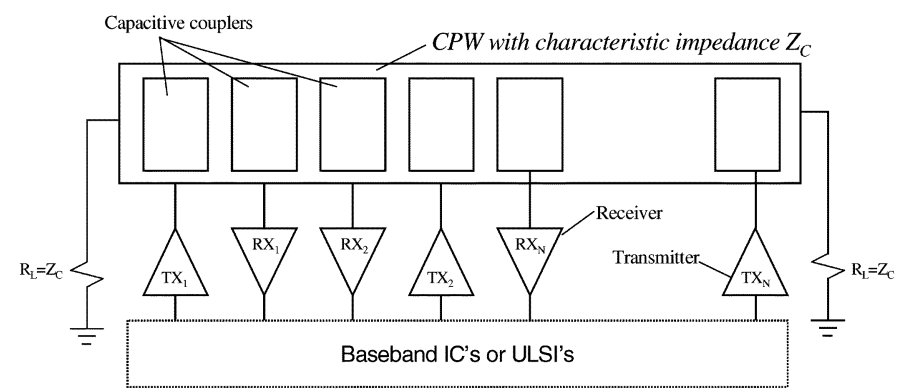

Fig. 22. Rrepresentative MCCDMA-I channel with multiple I/Os.

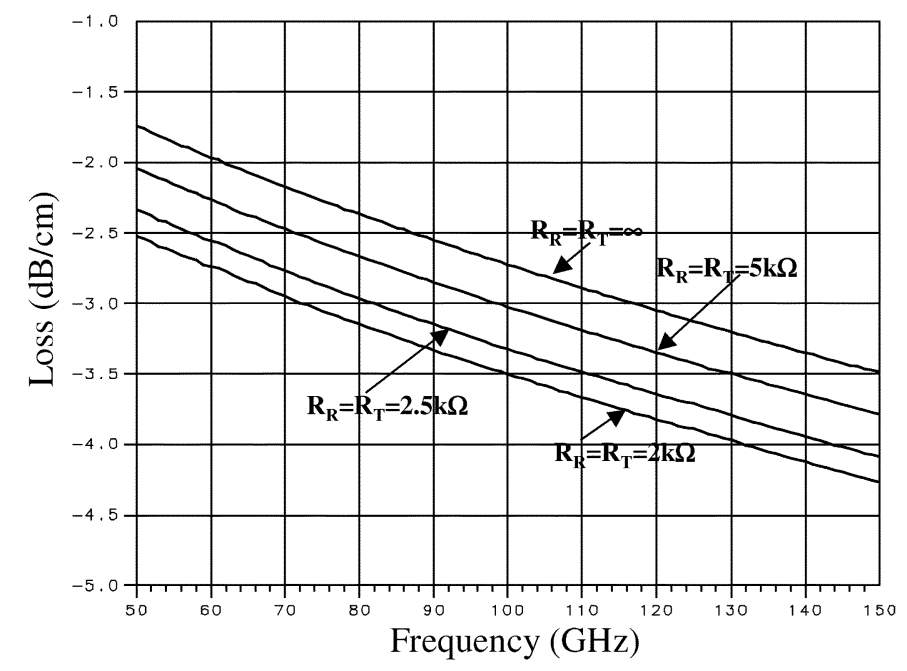

Fig. 23. Extra MTL attenuation caused by 20/20 shunted I/O transceivers.

alleviate the cross-channel interference. With orthogonal-coded and/or frequency-filtered RF transceivers, a passive MTL is suitable to relay ultra-broadband signals up to at least $150 \mathrm{GHz}$ [23].

Fig. 22 shows such a representative MCCDMA-I channel shunted by multiple I/Os through capacitor couplers. Since the channel is designed to hold bidirectional communications, both ends of the MTL are terminated with $Z_{C}$ to avoid the signal reflection. The transceiver's $\mathrm{I} / \mathrm{O}$ impedance $\left(\mathrm{R}_{\mathrm{T}}\right.$ and $\left.\mathrm{R}_{\mathrm{R}}\right)$ must be order of magnitude greater than $Z_{C}, 1 /\left(\omega \mathrm{C}_{\mathrm{T}}\right)$ and $1 /\left(\omega \mathrm{C}_{\mathrm{R}}\right)$ to preserve MTL's characteristic impedance $Z_{C}$ and achieve dispersion-free signal transmission. Simulations in Fig. 23 show insignificant signal attenuation $(0.3-0.8 \mathrm{~dB} / \mathrm{cm})$ and dispersion caused by 20/20 shunted I/O transceivers when choosing $\mathrm{R}_{\mathrm{T}}$ and $\mathrm{R}_{\mathrm{R}}$ as $2-5 \mathrm{k} \Omega$ and $\mathrm{C}_{\mathrm{R}}$ and $\mathrm{C}_{\mathrm{t}}$ on the order of $10 \mathrm{fF}$. Assuming the vertical coupling distance is $25 \mu \mathrm{m}$ and using polyimide $\left(\varepsilon_{\mathrm{r}}=4\right)$ as the dielectric between coupler electrodes, we estimate the pad size of $\mathrm{C}_{\mathrm{T}}$ or $\mathrm{C}_{\mathrm{R}}$ on the order $1000 \mu \mathrm{m}^{2}$. Capacitive couplers of this size can be easily implemented in ULSI. Further simulation based on full wave system analysis suggests that low loss and dispersion-free transmission over a broad-band $(>100 \mathrm{GHz})$ is achievable with SNR of $15 \mathrm{~dB}$ as calculated in Fig. 24 for reaching a low error $\left(\mathrm{BER}<10^{-14}\right)$ signal transmission, where the system is designed to hold five RF carrier-channels over the complete $100 \mathrm{GHz}$ and each carrier-channel covers 20-GHz band and contains four CDMA subchannels with 5-10 Gbps/subchannel, depending on the modulation scheme. Under such an arrangement, the $\mathrm{S} / \mathrm{N}$ budget for the intended RF-interconnect system 


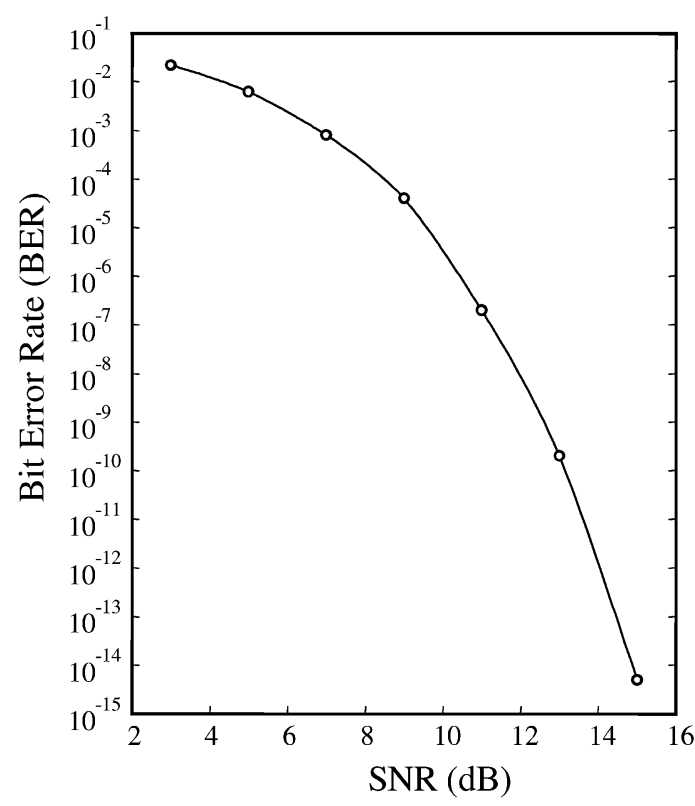

Fig. 24. BER versus SNR in MCCDMA-I with four I/O subchannels.

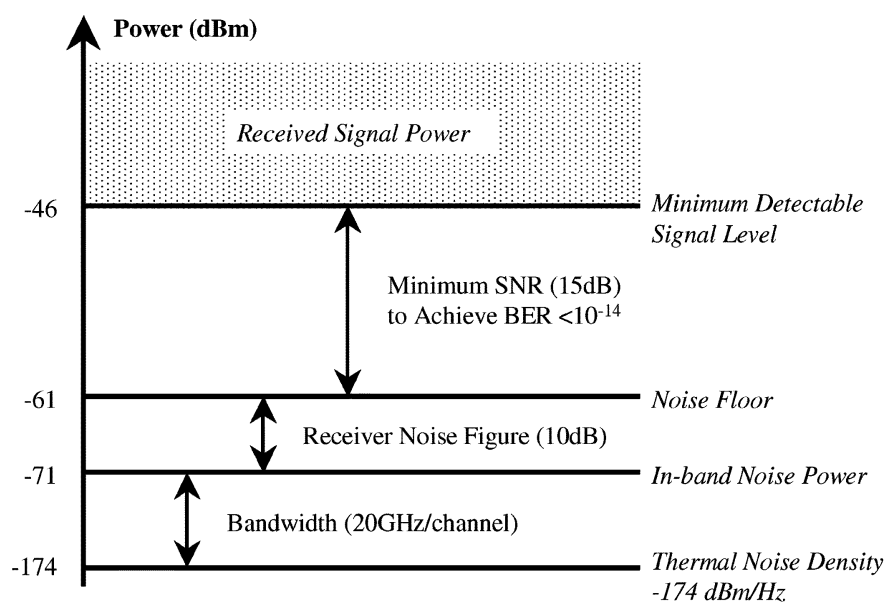

Fig. 25. Signal-to-noise budget for the intended MCCDMA-I system.

is calculated in Fig. 25 with a minimum detectable signal level of $-46 \mathrm{dBm}$ by assuming the receiver Noise Figure (NF) of $10 \mathrm{~dB}$.

Fig. 26 illustrates a schematic of 2-user MCCDMA-I system where each I/O port is allocated with different RF-carriers ( $f_{1}$ and $\mathrm{f}_{2}$ ). Within each carrier band the RF-modulated digital data is further mixed by using orthogonal Walsh codes to spread the spectrum. The first RF-modulated CDMA interconnect system has been demonstrated in [9].

\section{RINGS for SOC and SOP}

While the previous applications show the usage of SSCDMA-I for conventional CPU-memory communication and MCCDMA-I for wireless interconnect in SIP, the new interconnect technologies can also be used in low power embedded SOC. Examples are next-generation cell-phones, personal digital assistants (PDAs), portable game devices, etc. The trend is to merge multiple functions into one device, e.g., a cell phone with gaming and video capabilities, while at the same time

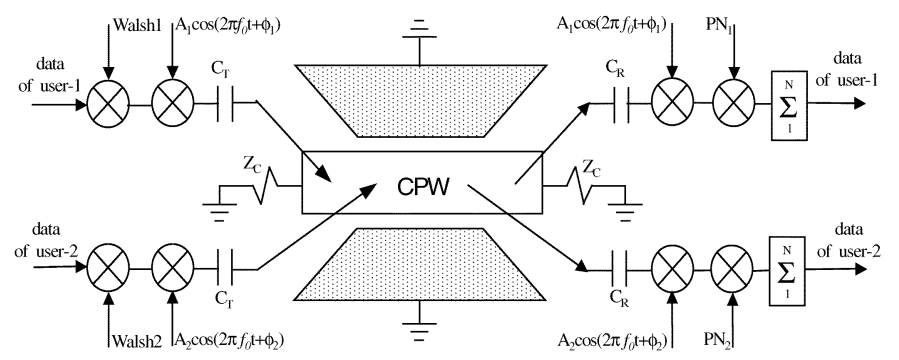

Fig. 26. Top-level schematic of a MCCDMA-I.

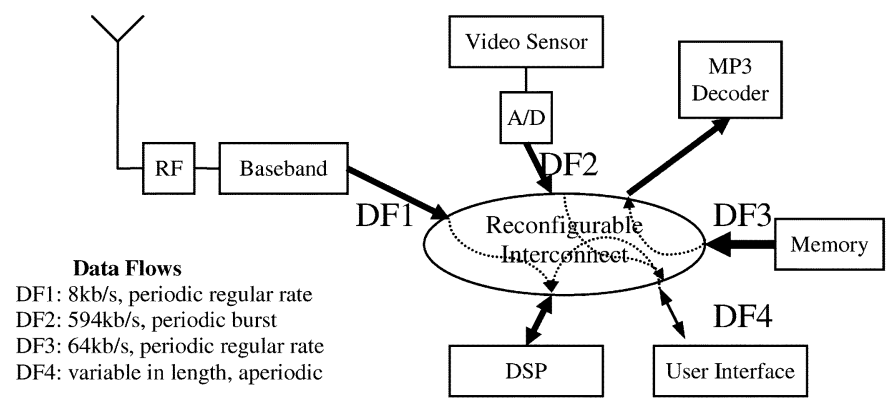

Fig. 27. Mobile phone system composed of heterogeneous cores in a RINGS architecture.

maintaining the battery life and reducing power consumption. The approach to combine performance and reduce energy is a SOC architecture with a heterogeneous mix of several dedicated cores, IP modules, embedded processors, etc. For instance, there will be a module for radio communication (e.g., 802.11 and Bluetooth and multiband cellular standards), DSP processors for wireless baseband communication, video and audio decoders, encryption, and security modules, and so on. Typical for the applications running on such a device is that the traffic patterns between the IP-cores are also heterogeneous in terms of different data rates, bursty, or regular, periodic, or sporadic, etc.

A typical example is illustrated in Fig. 27: it shows a multimedia mobile phone system on a RINGS platform [24]. Typical for a RINGS architecture is the co-existence of very different optimized modules, connected together by a re-configurable interconnect.

This example is used to illustrate that CDMA, FDMA, and TDMA interconnect can co-exist and provide the right communication channel for very different traffic flows, which have to co-exist on a single SOC or SIP. The CT-Bus (CDMA/TDMA bus), proposed in [25], integrates both CDMA and TDMA in a hierarchical structure and takes strengths from both of them. As shown in Fig. 28, a fixed amount of CDMA subchannels are separated by different spreading codes. Two or several subchannels can be grouped as one subchannel group. In Fig. 28, there are four CDMA subchannels, and they are divided into three different subchannel groups. The DF1 to DF4 illustrate data flows that need to be assigned to different subchannel groups. Because of the channel isolation feature of CDMA scheme, data flows on different subchannel groups will be well isolated without having impact on each other. For each CDMA subchannel-group, the data flows are further assigned to different TDMA time slots. 


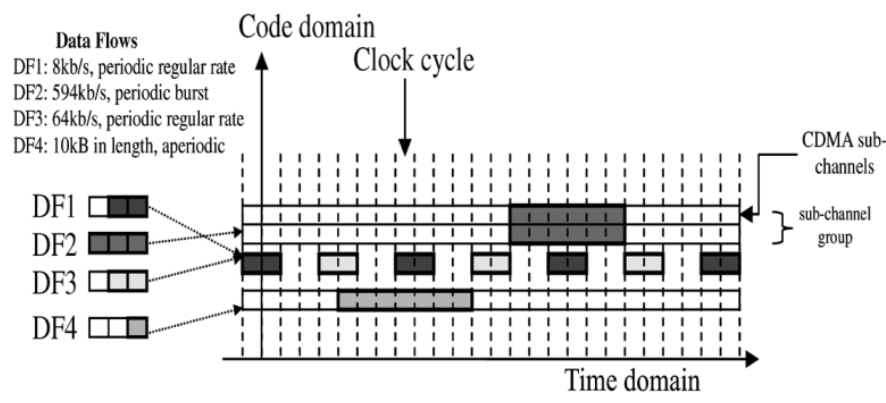

Fig. 28. Four CDMA subchannels divided into three different subchannel groups.

TABLE I

Data Flows on a Multimedia Mobile Phone.

\begin{tabular}{l|l|l|l|c|c}
\hline Data flows & \multicolumn{1}{|c|}{ Data rates } & Periodicity & $\begin{array}{c}\text { Traffic } \\
\text { Behavior }\end{array}$ & Priority & Remark \\
\hline DF1 & $8 \mathrm{~kb} / \mathrm{s}$ & Periodic & Regular & 2 & Signals from baseband to DSP \\
\hline DF2 * & $594 \mathrm{~kb} / \mathrm{s}$ & Periodic & Bursty & 1 & $\begin{array}{c}\text { Video data from video-sensor to } \\
\text { UI display (CIFF 176X144 pixels } \\
\text { format, 3 frames/s) }\end{array}$ \\
\hline DF3 & $64 \mathrm{~kb} / \mathrm{s}$ & Periodic & Regular & 2 & $\begin{array}{c}\text { MP3 decoder reads a music file } \\
\text { from memory }\end{array}$ \\
\hline DF4 & $10 \mathrm{kB}$ & Aperiodic & & 3 & $\begin{array}{c}\text { UI sends a MMS message file to } \\
\text { DSP }\end{array}$ \\
\hline \multicolumn{5}{c}{ * Note: Each burst of DF2 cannot be delayed more than 0.2 second }
\end{tabular}

Reallocation of the traffic flows to subchannel groups can be achieved by simply re-assigning the spreading codes to traffic flows, enabling the reconfiguration of the CT-Bus.

Table I specifies four data flows as similar to the mobile phone system mentioned previously. The CT-Bus has a total bandwidth of $1 \mathrm{Mb} / \mathrm{s}$, and supports four subchannels. Three subchannel groups have been created with a bandwidth of $512 \mathrm{kbps}$, $256 \mathrm{kbps}$, and $256 \mathrm{kbps}$. The regular data flows, DF1 and DF3, are assigned to a dedicated $256 \mathrm{kbps}$ subchannel. DF2 is assigned to the $512 \mathrm{kbps}$ subchannel. DF3 with a periodic data is assigned to another $256 \mathrm{kbps}$ subchannel.

As a comparison, the same data flow patterns have been applied to a WFQ (Weighted-Fair-Queuing) TDMA bus with the bandwidth of 1 Mbps. The arbiter of WFQ-TDMA bus uses block mode, which will transmit the DF with the highest priority. Time slots will be assigned in proportion to the average data rates if data flows have the same priority.

We run simulations of the CT-Bus and the WFQ-TDMA bus for a period of one second and record the average latencies of data flows. Fig. 29 shows the average latencies of the data flows on WFQ-TDMA bus. DF2 has the highest priority and has three periodic bursts in a second. It is obvious that the regular data flows DF1 and DF3 have been influenced by the burst data flows DF2 with higher priority. Fig. 30 shows the average latency of the data flows on the CT-Bus. Because DF1 and DF3 have their own subchannel, the maximum latency is less than 100 us and the burst traffic from DF2 has no interference. The average latency of DF2 is twice as large as in WFQ-TDMA. However, DF2 can still meet its requirement, each burst has delay no more than 0.2 second.

The three examples in this section clearly indicate that nextgeneration systems can no longer be using TDMA busses alone. Interesting future research work will include modeling the new interconnect technologies such that they can be used into architecture and system design.

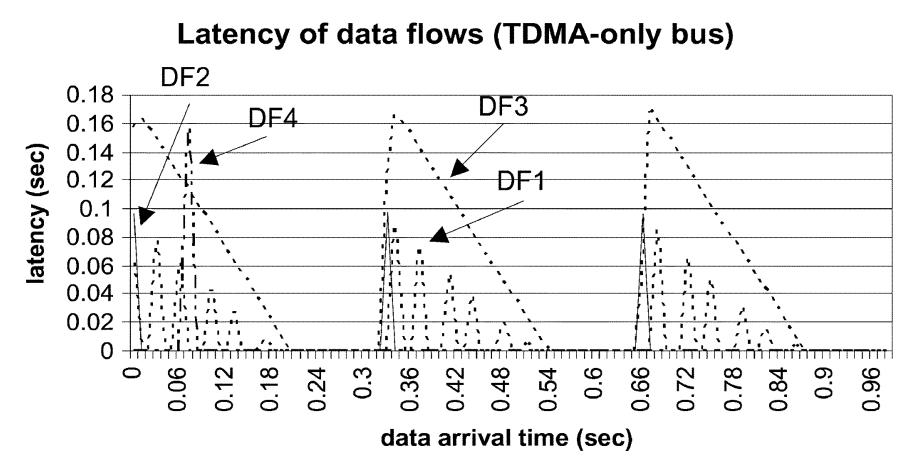

Fig. 29. Latency of data flows on the WFQ-TDMA bus.

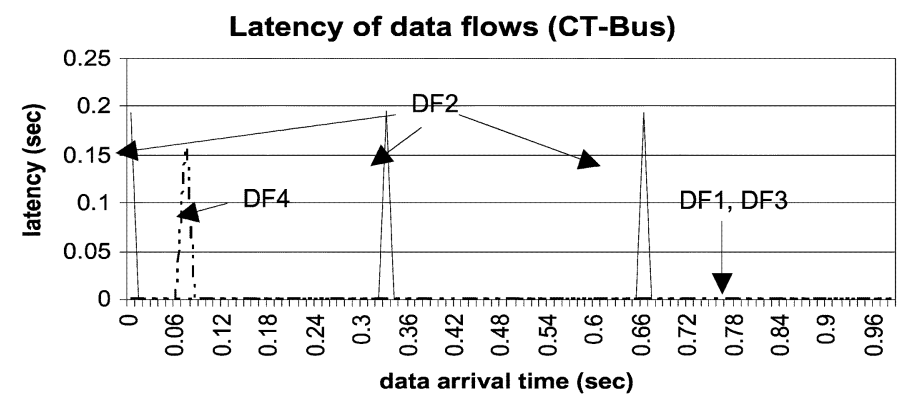

Fig. 30. Latency of data flows on the CT-Bus.

\section{SYSTEM BENEFITS}

With the feature size of CMOS shrinking below $90 \mathrm{~nm}$, many complex data processing and function features that previously could not be integrated on a single chip due to either large area or high speed can now be successfully implemented, as evidenced by products that are emerging with complete SOC and/or SIP. The major challenge in pushing the frontiers of system integration further in the next decade or more lies in low-cost, low-power interconnect technology needed to provide the necessary aggregate data rate, re-configurability, and latency required by chips or system of chips with every increasing complexity. In this paper, we have reviewed several new interconnect schemes that can potentially meet this challenge and will now discuss their benefits to the performance of next-generation ULSI systems.

1) Aggregate Data Rate: Several research efforts on interand/or intra-chip high-speed interconnects have been pursued in the recent past. For example, high-speed end-to-end wire interconnects (with signal-processing hardware, such as, channel equalizers, at both ends) for data transmission speeds up to 4-10 Gbps have been successfully demonstrated [4], [18]. However, such wire interconnect is often based on TDMA-I. To compensate for the signal loss over the wire, such systems would require sophisticated equalizers to compensate for frequency dependent attenuation. In particular, since the I/O data rate equals the aggregate data rate and cannot be separately controlled in a system, the aggregate data rate is likely to be constrained to $40 \mathrm{Gbps} / \mathrm{pin}$ even in 65-nm CMOS technology (limited by $\mathrm{f}_{\mathrm{t}} / 5$ of the CMOS technology [26], [27], where $\mathrm{f}_{\mathrm{t}}=200 \mathrm{GHz}$ forecasted by ITRS [28]). In contrast, our proposed interconnect schemes based on CDMA and FDMA can achieve aggregate data rate beyond 100 
Gbps/pin given the availability of the same $65 \mathrm{~nm}$ technology (in principle, the maximum carrier frequency could approach $\mathrm{f}_{\mathrm{t}} / 2$ ). This is because in both CDMA and FDMA, the I/O data rate can be reduced by increasing $N_{c}$ and $N_{f}$, respectively. For example, given a MCCDMA-I scheme with $N_{f}$ of 32 channels, $N_{c}$ of 4 , and an aggregate data rate of $128 \mathrm{Gbps} / \mathrm{pin}$, each I/O then will run at $1 \mathrm{Gbps}$. At this speed and with four codes, the correlator required for each $\mathrm{I} / \mathrm{O}$ can be implemented in less than $1000 \mu \mathrm{m}^{2}$ while dissipating less than $1 \mathrm{~mW}$ in $65-\mathrm{nm}$ CMOS technology [7], [28].

2) Concurrent Multil/O Services: As more systems become more heterogeneous, a variety of data sources with different latency constraints and/or priority must be communicated between various subsystems. It becomes important to provide concurrent multiI/O services to these subsystems so as to reduce latency as discussed in two examples mentioned earlier in DRAM and RINGS. Using FDMA and/or CDMA, one can easily provide concurrent multiI/O services, while maintaining reliability. Another potential benefit of multiI/O services to a complex SOC is that it may enable the architect to cut down the number of I/O ports on the chip, since one can use FDMA-I together with CDMA-I to interleave multiple data sets on the network, one I/O port can serve multiple $\mathrm{I} / \mathrm{O}$ needs simultaneously.

3) Re-Configurability: As systems become more versatile, next-generation devices may become realtime configurable into different functions. For instance, the next-generation smart phone can morph into a portable digital television, DVD player, or handheld computer. The architectural flexibility that is being increasingly demanded by such a device is the ability to reconfigure any given subsystem and interconnections in realtime. In our system, CDMA-I, FDMA-I, or MCCDMA-I can allow each I/O pair to choose an orthogonal address code and/or frequency channel. The address code can be electronically changed for interconnect reconfiguration on-the-fly.

\section{SUMMARY}

Future ULSI interconnect systems, either in SOC or SIP, demand not only to be extremely fast but also to be re-configurable and must be capable to provide bidirectional and multiI/O services. In this paper, we have reviewed recently developed advanced $\mathrm{RF} / \mathrm{b}$ aseband interconnects that can be further developed to satisfy all above system needs. Unlike traditional wired interconnects based only on TDMA-I for data transmission, these new interconnect schemes allow using additional modern multiple access algorithms including CDMA-I, FDMA-I, SCRFI, and MCCDMA-I and their derivatives to boost the bandwidth, the channel concurrency and at the same time reduce the channel latency. The physical transmission media is also no longer limited to the direct-coupled metal wire. It can be capacitor-coupled and/or through any gufoaided/free transmission media. The capacitor-coupled SCRFI can also play an important role in future 3-D IC by eliminating the costly vertical metal interconnects. The continuous development of the new interconnect schemes would inevitably change the scope of ULSI data communication structure and impact the next-generation computer system design.

\section{ACKNOWLEDGMENT}

The authors are grateful to contract supports of U.S. DARPA and SRC throughout the development of advanced RF-interconnects.

\section{REFERENCES}

[1] K. C. Saraswat and F. Mohammadi, "Effect of interconnection scaling on time delay of VLSI circuits," IEEE Trans. Electron Devices, vol. ED-29, pp. 645-650, Apr. 1982.

[2] J. D. Meindl, "Integration limits on 21st century gigascale integration," in IEEE Interconnect Technology Conf.-Short Course, San Francisco, CA, May 31, 1998.

[3] M. T. Bohr and Y. A. El-Mansy, "Technology for advanced high performance microprocessors," IEEE Trans. Electron Devices, vol. 45, pp. 620-625, Mar. 1998.

[4] J. L. Zerbe, C. W. Werner, V. Stojanovic, F. Chen, J. Wei, G. Tsang, D. Kim, W. F. Stonecypher, A. Ho, T. P. Thrush, R. T. Kollipara, M. A. Horowitz, and K. S. Donnelly, "Equalization and clock recovery for a 2.5-10-Gb/s 2-PAM/4-PAM backplane transceiver cell," IEEE J. SolidState Circuits, vol. 38, pp. 2121-2130, Dec. 2003.

[5] K. Farzan and D. A. Johns, "A CMOS 10-gb/s power-efficient 4-PAM transmitter," IEEE J. Solid-State Circuits, vol. 39, pp. 529-532, Mar. 2004.

[6] R. Farjad-Rad, C.-K. K. Yang, and M. A. Horowitz, "A 0.3- $\mu \mathrm{m}$ CMOS 8-Gb/s 4-PAM serial link transceiver," IEEE J. Solid-State Circuits, vol. 35, pp. 757-764, May 2000.

[7] M. F. Chang et al., "RF/wireless interconnect for inter- and intra-chip communications," Proc. IEEE, vol. 89, no. 4, Apr. 2001.

[8] M. F. Chang, H. Shin, and L. Zhang, "RF-interconnect for future interand intra-ULSI communications," in IEDM Tech. Dig., Dec. 2001, pp. 23.4.1-23.4.4.

[9] H. Shin, Z. Xu, and M. F. Chang, "RF-interconnect for multiGb/s digital interface based on $10 \mathrm{GHz}$ RF-modulation in $0.18 \mu \mathrm{m} \mathrm{CMOS,"} \mathrm{in}$ MTT-S Dig., vol. 1, Jun. 2002, pp. 477-480.

[10] Z. Xu, H. Shin, J. Kim, M. F. Chang, and C. Chien, "Giga bit/s CDMAinterconnect transceiver chip-set with multilevel signal data recovery for re-configurable VLSI system," in Proc. IEEE Int. Solid-State Circuits Conf. Tech. Dig., San Francisco, CA, Feb. 2003, pp. 322-323.

[11] J. Kim, Z. Xu, and M. F. Chang, "A 2-Gbps/pin source synchronous CDMA bus interface with simultaneous multichip access and reconfigurable I/O capability," in Proc. IEEE Custom Integrated Circuits Conf. (CICC), Sept. 2003, pp. 317-320.

[12] - "Reconfigurable memory bus systems using multiGbps/pin CDMA I/O transceivers," in Proc. IEEE Int. Symp. Circuits and Systems (ISCAS), vol. 2, May 2003, pp. II-33-II-36.

[13] Q. Gu, Z. Xu, J. Kim, J. Ko, and M. F. Chang, "Three-dimensional circuit integration based on self-synchronized RF-interconnect using capacitive coupling," in 2004 Symp. VLSI Technology and Circuits (VLSI), HI, June 2004, pp. 96-97.

[14] CDMA Systems Engineering Handbook, J. S. Lee and L. E. Miller, Eds., Artech House, Norwell, MA, 1998.

[15] R. Farjad-Rad et al., "A 0.3- $\mu$ m CMOS 8-Gb/s 4-PAM serial link transceiver," in Proc. Symp. VLSI Circuits, May 1999.

[16] A. Martin et al., " $8 \mathrm{~Gb} / \mathrm{s}$ differential simultaneous bidirectional link with $4 \mathrm{mV} 9$ ps waveform capture diagnostic capability," in IEEE Int. SolidState Circuits Conf. Tech. Dig., Feb. 2003, pp. 478-479.

[17] A. P. Chandrakasan et al., Design of High Performance Microprocessor Circuits. New York, 2001, pp. 352-376.

[18] J. Ko, Z. Xu, Q. Gu, C. Chien, and M. F. Chang, "A simultaneous $\mathrm{RF} /$ base-combined multiband bi-directional interconnect for inter-chip link/bus system applications," IEEE J. Solid-State Circuits, submitted for publication.

[19] A. Rahman et al., "Comparison of key performance metrics in two- and three-dimensional integrated circuits," in Proc. IEEE Interconnect Tech. Conf., Jun. 2000, pp. 157-159.

[20] M. B. Kleiner, S. A. Kuhn, and W. Weber, "Modeling the impact of 3-D technology on the performance of the memory hierarchy of RISC systems," in Proc. ISCAS'95, vol. 3, 1995.

[21] R. Uscola and M. Tutt, "Determining the inductance of a through-substrate via using multiple on-wafer test approaches," in Proc. IEEE 2001 Int. Conf. Microelectronic Test Structures, vol. 14, Mar. 2001. 
[22] H. Chow and I. Wang, "High performance automatic gain control circuit using a S/H peak-detector for ASK receiver," in Proc. Int. Conf. on Electronics, Circuits, and Systems, vol. 2, Sep. 2002.

[23] M. Y. Frankel et al., "Terahertz attenuation and dispersion characteristics of coplanar transmission lines," IEEE Trans. Microwave Theory Tech., vol. MTT-39, no. 6, Jun. 1991.

[24] I. Verbauwhede and M. F. Chang, "Reconfigurable interconnect for next generation systems," in 2002 ACM Int. Workshop on System Level Interconnect Prediction (SLIP 2002), Apr. 2002, pp. 71-74.

[25] B. C. Lai, P. Schaumont, and I. Verbauwhede, "A heterogeneous CDMA/TDMA bus for future SOC," in Proc. 38th Annu. Asilomar Conf. Signals, Systems, and Computers.

[26] T. H. Hsu and P. Gray, "A monolithic 480 Mbps parallel AGC/decision/clock-recovery circuit in 1.2 um CMOS," IEEE J. Solid State Circuits, vol. 28, no. 12, Dec. 1993.

[27] S. K. Enam and A. A. Abidi, "NMOS IC's for clock and data regeneration in gigabit-per-second optical fiber receiver," IEEE J. Solid State Circuits, vol. 27, no. 12, pp. 1763-1774, Dec. 1991.

[28] (2003) RF and analog mixed-signal technologies for wireless communication. International Technology Roadmap for Semiconductors (ITRS). [Online] Available: http://public.itrs.net/Files?2003ITRS/ Home2003.htm

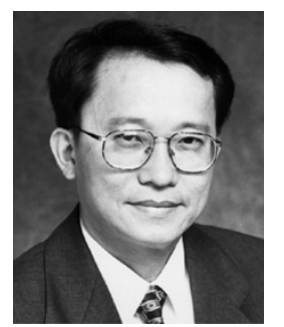

Mau-Chung Frank Chang (F'96) received the B.S. degree in physics from National Taiwan University, Taipei, Taiwan, R.O.C., in 1972, the M.S. degree in material science from National Tsing-Hua University, Hsinchu, Taiwan, in 1974, and the Ph.D. degree in electrical engineering from National Chiao-Tung University, Hsinchu, in 1979.

He is currently a Full Professor and the Vice Chair of the Electrical Engineering Department, University of California at Los Angeles (UCLA). Before joining UCLA, he was the Assistant Director and Department Manager of the High Speed Electronics Laboratory, Rockwell Science Center, Thousand Oaks, CA, during 1983-1997. In this tenure, he successfully developed and transferred the AlGaAs/GaAs HBT technology form the research laboratory to the production line (Conexant and Skyworks). The HBT production has now grown into a multibillion dollar business worldwide. He was also the Principal Investigator at Rockwell to lead the U.S.-DARPA ADC and DAC development for direct conversion transceiver (DCT) and digital radar receivers (DRR) systems. During his career, his research work has been mostly in the development of high-speed semiconductor devices and integrated circuits for mixed signal communication and radar system applications. He was the inventor of the multiI/O, re-configurable RF/wireless interconnects based on FDMA and CDMA multiple access algorithms for inter- and intra-ULSI communications. He was also pioneered in demonstrating the first dual mode (CDMA/AMPS) RF power amplifier based on Si/SiGe HBTs; the first K-band $(24-27 \mathrm{GHz})$ RFIC in CMOS and the first single-chip CMOS ADC with 1-GHz instantaneous bandwidth. He has served as a Board Director in two GaAs manufacturing companies, including GCS, Torrance, CA, and GCTC, Hsinchu, Taiwan. Both GCS and GCTC are regarded as world-class pure-player foundry houses for microwave and millimeter-wave integrated circuits (MMIC) productionHe has authored or co-authored over 200 technical papers, ten book chapters, edited one book, and held 20 U.S. patents.

Dr. Chang is a Co-Editor of the IEEE TRANSACTIONS ON ELECTRON DEVICES and was a Guest Editor for the IEEE JOURNAL OF SOLID-STATE CIRCUITS in 1991 and 1992, respectively, and the Journal of High-Speed Electronics and Systems in 1994. He was honored with Rockwell's Leonardo Da Vinci (Engineer of the Year) award in 1992, National Chiao-Tung University's Distinguished Alumni Award in 1997, and National Tsing-Hua University's Distinguished Alumni Award in 2002. He was named an IEEE Fellow in 1996 for his pioneering contributions in ultra-high-speed HBT integrated circuit development. Since 1997, he has founded a communication chip-design company (G-Plus, Westwood, CA, recently acquired by SST).

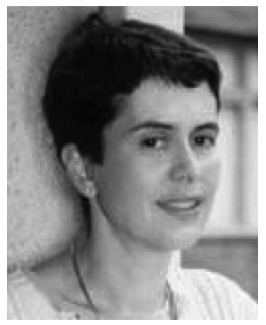

Ingrid Verbauwhede (M'93-SM'00) received the electrical engineering degree in 1984 and the Ph.D. degree in applied sciences from the Katholieke Universiteit (KU) Leuven, Leuven, Belgium, in 1991.

She was a Lecturer and Visiting Research Engineer at the University of California at Berkeley from 1992 to 1994. From 1994 to 1998, she was a Principal Engineer, first with TCSI and then with Atmel, Berkeley, CA. She joined the University of California at Los Angeles in 1998 as an Associate Professor at the KU Leuven in 2003. Her interests include circuits, processor architectures and design methodologies for real-time embedded systems in application domains such as cryptography, digital signal processing, wireless, and high-speed communications.

Dr. Verbauwhede is or was a member of several program committees, including DAC, ISSCC, DATE, CHES, ICASSP. She is currently the Design Community Chair of the 42nd DAC Executive Community.

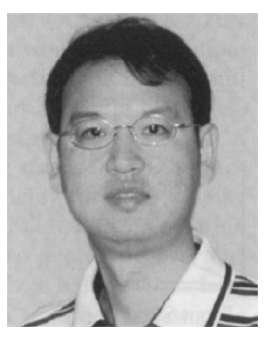

Charles Chien (M'00) received the B.S.E.E. degree from the University of California at Berkeley and the M.S. and Ph.D. degrees from the University of California at Los Angeles (UCLA).

$\mathrm{He}$ is the Vice President of Communication Systems and IC at SST Communications, Los Angeles, CA. He is also an Assistant Adjunct Professor at UCLA. He has previously worked at Bell Communications Research on a digital SONET-like HDTV system and at Rockwell Science Center, Thousand Oaks, CA, on broadband wireless multimedia networks and low-power wireless sensor networks. His interests are mainly in the design of system-on-chip solutions for wireless multimedia and networking applications. He has published in various journals and conferences, and has authored the book Digital Radio Systems on a Chip (Norwell, MA: Kluwer, 2001).

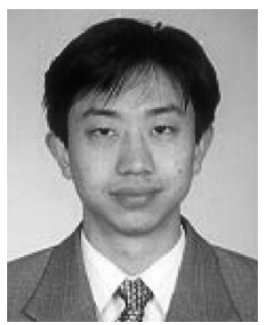

converters.
Zhiwei Xu received the M.S. degree in electrical engineering from Fudan University, Fudan, China, in 2000 and the Ph.D. degree from the Electrical Engineering Department, University of California at Los Angeles, in 2003.

He then joined G-Plus Inc., which was merged into SST Communication Corporation, to develop a wireless LAN transceiver chip. His research interests include various CMOS high-speed circuits for wired/wireless communication systems, integrated CMOS RF IC design, and low-power AD/DA

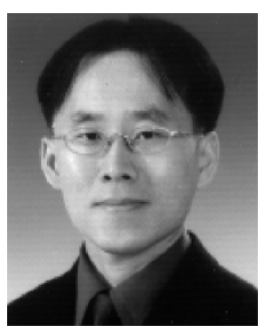

Jongsun Kim (S'01) received the B.S. degree in electronics engineering from Hanyang University, Seoul, Korea, in 1992 and the M.S. degree in electrical engineering from Pohang University of Science and Technology (POSTECH), Pohang, China, in 1994. He is currently working toward the Ph.D. degree in the field of integrated circuits and systems at the University of California at Los Angeles (UCLA).

From 1994 to 2001, he was with Samsung Electronics as a Senior Research Engineer and a Manager in the DRAM Design Group, where he worked on the design and development of synchronous DRAMs, SGDRAMs, Rambus DRAMs, and other specialty DRAMs. Since 2001, he has been with the Electrical Engineering Department, UCLA. His research interests are in the area of high-performance mixed-signal circuits and systems design. His current area of research includes reconfigurable RF/CDMA/FDMA interconnect circuits and systems, high-speed, and low-power I/O transceiver circuits for chip-to-chip communications, clock recovery circuits (PLLs/DLLs), and high-performance reconfigurable memory circuits and systems. He holds seven U.S. patents in the field of electronic circuits. 


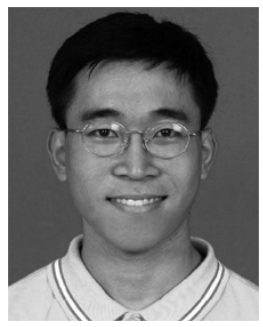

interface circuits.

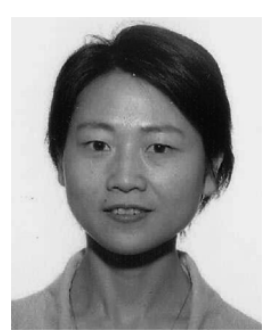

Jenwei Ko received the B.S. and M.S. degree in mechanical engineering from National Taiwan University, Taipei, Taiwan, R.O.C., in 1994 and 1996, respectively. He is currently pursuing the Ph.D. degree in the field of integrated circuits and systems at the University of California at Los Angeles.

Since 2001, he has been with the Electrical Engineering Department, UCLA. His research interests include analog and mixed-signal circuits and system design, CMOS communication circuits and system design, RF-interconnect circuits, and high-speed I/O

Qun Gu received the B.S. degree from Huazhong University of Science and Technology, Wuhan, China, in 1997 and the M.S. degree from the University of Iowa, Iowa City, in 2002. She is currently pursuing the Ph.D. degree at the University of California at Los Angeles.

Her research interests include RF interconnect for three-dimensional ICs and very high-speed transceiver circuits, especially transmitter-side circuit design.

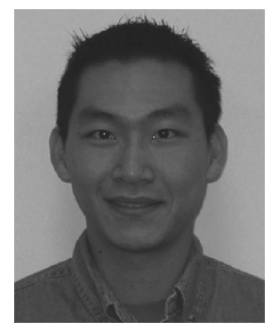

Bo-Cheng Lai received the B.S. degree from the Electronics Engineering Department, National Chiao-Tung University, Hsinchu, Taiwan, R.O.C., in 1999 and the M.S. degree in electrical engineering department from the University of California at Los Angeles in 2003, where he is currently pursuing the Ph.D. degree.

His research concerns the design of interconnect architectures for system-on-chip, multiprocessor architectures, networking-embedded systems, and security protocols of sensor networks.

Mr. Cheng is the recipient of Henry Samueli Fellowships for fall 2002, he also received the scholarship from John Deere Foundation in 2003. He is a student member of IEEE. 